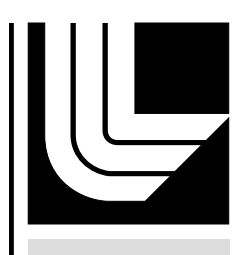

LAWRENCE LIVERMORE NATIONAL LABORATORY

Linear Permittivity Tapering in a Cerenkov Microwave Source with a Pre-Bunched Beam

B. R. Poole, J. R. Harris

February 22, 2013

Physics of Plasmas 
This document was prepared as an account of work sponsored by an agency of the United States government. Neither the United States government nor Lawrence Livermore National Security, LLC, nor any of their employees makes any warranty, expressed or implied, or assumes any legal liability or responsibility for the accuracy, completeness, or usefulness of any information, apparatus, product, or process disclosed, or represents that its use would not infringe privately owned rights. Reference herein to any specific commercial product, process, or service by trade name, trademark, manufacturer, or otherwise does not necessarily constitute or imply its endorsement, recommendation, or favoring by the United States government or Lawrence Livermore National Security, LLC. The views and opinions of authors expressed herein do not necessarily state or reflect those of the United States government or Lawrence Livermore National Security, LLC, and shall not be used for advertising or product endorsement purposes. 


\title{
Linear permittivity tapering in a Cerenkov microwave source with a pre-bunched beam
}

\author{
B. R. Poole ${ }^{1, a)}$ and J. R. Harris ${ }^{2}$ \\ 1) Lawrence Livermore National Laboratory, Livermore, CA 94550 USA \\ ${ }^{2)}$ Dept. of Electrical and Computer Engineering, Colorado State University, \\ Fort Collins, CO 80523 USA
}

(Dated: 16 February 2013)

Cerenkov microwave sources use a dielectric-lined waveguide to reduce the velocity of the electromagnetic wave and provide efficient energy transfer between the wave and the driving electron beam. Tapering the permittivity of the dielectric to maintain synchronism between the beam and the wave as the beam loses energy can increase the efficiency of these devices. Here we consider such a structure driven by an electron beam with a harmonic density perturbation. Particle-In-Cell (PIC) simulations and a macro-particle model based on the slowly varying envelope approximation are first used to examine an un-tapered baseline case. PIC simulations of the source with linear tapers over the entire amplifier length as well as over only a section of the amplifier where the beam executes synchrotron oscillations are examined. The efficiency for the baseline un-tapered source is $18 \%$, while efficiencies up to approximately $48 \%$ are found using a taper in dielectric permittivity. Results of the best performing cases are presented. Detailed examination of longitudinal phase space, particle energy distributions, evolution of longitudinal wavenumber, and phase dynamics are presented from the PIC simulations.

PACS numbers: 41.60.Bq, 41.60.Cr

a)Electronic mail: poole1@llnl.gov 


\section{INTRODUCTION}

When electrons travel through a region with velocities exceeding the local phase velocity of light, electromagnetic energy will be released in the form of Cerenkov radiation. The possibility of using this process for generating microwave radiation was first proposed by Ginsburg $^{1}$ in 1947. However, the Cerenkov mechanism by itself is a relatively inefficient source of microwave energy due to its continuous spectrum and unfavorable scaling of emitted power with frequency. To overcome these limitations, Cerenkov microwave sources use a combination of electron beam bunching and resonant structures which serve to generate coherent radiation concentrated at discrete wavelengths. ${ }^{2,3}$ These basic features can give rise to devices with different physical configurations and modes of operation. One common configuration is a dielectric-lined waveguide, a structure which supports TM modes whose axial phase velocity is slowed by the presence of the dielectric. When an electron beam is traveling through the structure at a velocity somewhat larger than one of these modes, synchronism will occur between the beam's slow space charge wave and the TM mode, supporting the Cerenkov interaction. These modes provide an axial electric field, causing the beam to bunch at the resonant frequency and the radiation to become coherent. Because the TM modes are maximum near the dielectric-vacuum interface, the interaction can be enhanced by placing most of the beam current near that interface. This generally requires that the beam be guided by a strong axial magnetic field. For Cerenkov sources of this type, the beam energy is typically between several hundred $\mathrm{keV}$ and $1 \mathrm{MeV}$. In this energy range, the beam is sufficiently relativistic that significant amounts of beam energy can be extracted before its velocity reduces to a point where it is no longer synchronous with the TM mode, but not so relativistic as to prevent the bunching required for coherent emission.

The nature of the radiation generated from such a system depends on how the system is excited, and on the details of the beam bunching process. One class of mechanisms produce gain as described above by allowing the TM mode to drive a beam density modulation. When a continuous electron beam is transported through the structure, the Cerenkov instability will allow bunching and gain at a frequency defined by the beam-mode synchronism. The system will therefore amplify microwave signals at that frequency which are present as noise

or are deliberately introduced into the structure. ${ }^{4-6}$ The picture is more complicated when the beam initially contains density perturbations such as the steep rise in current at the 
head of a pulsed beam. ${ }^{7}$ These perturbations excite the structure's TM modes over a range of frequencies, not just the design operating frequency. This drives the formation of a short-pulse wave packet. Because the group velocity of these modes is slower than their phase velocity, this wave packet will slip backwards in the beam frame, continually sampling "fresh" regions of the beam from which power can be extracted.

A different type of interaction occurs if the injected beam consists of bunches that are much shorter than the operating wavelength, as would be the case when driven by a photoinjector. ${ }^{8}$ If the bunches are injected into a structure without a pre-existing microwave field, superradiant emission will occur without gain. ${ }^{9}$ If a pre-existing microwave field is present, its longitudinal electric field can be used to accelerate or decelerate the pre-bunched beam. ${ }^{10-13}$ In this operating mode, the system resembles a conventional RF accelerator.

Cerenkov sources are highly flexible sources of microwave energy. They can be tuned over a wide frequency band, exhibit relatively high efficiency, and can operate at high power. ${ }^{14}$ Their principle disadvantage is the potential for beam-driven charging of the dielectric and subsequent dielectric failure. ${ }^{15}$

When the Cerenkov source is operated as an amplifier, a TM mode grows as it extracts energy from the beam. Because the beam is not highly relativistic, its velocity decreases. This, in turn shifts the synchronous condition or operating point away from the design frequency, leading to saturation at that frequency. If the dielectric loading were then changed to reduce the phase velocity of the mode and to shift the synchronous point back to the design frequency, the amplification process could continue, and additional energy would be extracted from the beam and put into the mode at that frequency. This process is known as tapering the dielectric. ${ }^{16}$ Ideally, the waveguide parameters would be continuously modified, so that the synchronous point would continue to stay at the desired operating frequency as the beam energy decreases.

Tapering in Cerenkov sources can be accomplished in two general ways. The first is to change the mode phase velocity in the structure by gradually changing the fraction of the structure filled with dielectric. The simplest way of doing this is by gradually changing the dielectric thickness. This was considered by Gore, Asgekar, and Sen, ${ }^{17}$ who performed numerical studies of a $2.05 \mathrm{MeV}$ sheet beam propagating above a conductive plane having a dielectric film coating designed to operate at $350 \mathrm{GHz}$. They used a dielectric layer thickness 
which was initially constant until the point at which beam energy loss was first seen in untapered cases, and then decreased the thickness with a quadratic or cubic dependence on distance. By doing this, they were able to increase the extraction efficiency by over a factor of two. They did not find that tapering affected the requirements on beam energy spread. The impact of variations in the thickness of a dielectric-lined waveguide Cerenkov source was also considered by van der Slot, de la Fuente, and Boller. ${ }^{18}$ They were primarily concerned that an unwanted, random variation in dielectric liner thickness would randomly detune the system from the synchronous operating condition. However, their mathematical approach also enabled them to study the impact of a linear taper in dielectric thickness, applied to the case of a Cerenkov source designed for operation at $50 \mathrm{GHz}$, using a $100 \mathrm{keV}, 800 \mathrm{~mA}$ beam. They found that such a linear taper increased the power extraction from the beam, with stronger tapers providing better extraction rates, limited only by the point at which the constant radius beam was intercepted by the dielectric liner. The volume occupied by the dielectric can also be varied by keeping its thickness constant, while machining grooves into the dielectric, effectively "derating" the dielectric's permittivity by introducing vacuum inclusions. Tapering can be accomplished by adjusting the width or depth of these grooves. This approach was investigated by Shiozawa's group at Osaka University. ${ }^{19,20}$ They simulated a parallel plate waveguide with a grooved dielectric sheet applied to the inside of one of the conductors, and propagated an ion-neutralized electron beam (energies $443 \mathrm{keV}$ and $533 \mathrm{keV}$ ) between the dielectric sheet and the opposite conductor. Dielectric and waveguide properties were chosen for Cerenkov radiation at $134 \mathrm{GHz}$ or $126 \mathrm{GHz}$, and to suppress the Smith-Purcell instability. ${ }^{21}$ Electromagnetic wave scattering from the grooves was neglected. Their simulation was divided into steps, with the groove width or depth reduced iteratively to track the reduction of beam energy and to maintain the synchronous condition at the operating frequency. By optimizing the dielectric, their device efficiency was increased from $15 \%$ to $35 \%$. The limitation on this approach occurs when the groove width or depth is reduced to zero, at which point the grooved dielectric sheet becomes a smooth sheet.

The second approach to tapering Cerenkov sources is to change the mode phase velocity by gradually changing the dielectric constant. This has been investigated by several researchers. Schächter and Nation ${ }^{12,13}$ considered the case of an $850 \mathrm{keV}$ electron beam propagating at the center of a dielectric-lined cylindrical waveguide designed to produce radiation at $8.75 \mathrm{GHz}$. With a pre-bunched beam, they considered two types of tapers, one 
using a linear dependence of permittivity with position, and the other using a taper which ensured the mode phase velocity tracked the average beam velocity to maintain synchronism at the design frequency. When operated as an accelerator, the latter tapering accelerated the beam to an energy twice that with the linear taper, while virtually no acceleration was seen for a uniform dielectric without tapering. They also considered an un-bunched beam, and optimal linear tapering increased the device efficiency from $5 \%$ to $30 \%$. Tapering of the permittivity in planar Cerenkov sources was considered by Gore, Asgekar, and Sen, ${ }^{17}$ who found it gave results comparable to tapering the dielectric thickness. Shiozawa and Yoshitake $^{22}$ also performed numerical studies of varying the permittivity using a parallel plate waveguide, slab dielectric, and ion-neutralized sheet beam system, similar to that used for the grooved dielectric studies. However, they used a Kerr-like dielectric with properties chosen to "automatically" adjust the dielectric constant in response to the growth of the TM mode, enabling them to approximately double the peak extracted beam energy. Hirata and Shiozawa ${ }^{14}$ improved the performance of this system using conventional dielectrics, while using a more nuanced optimization method. Rather than increasing the dielectric constant to reduce the phase velocity, they increased or decreased the dielectric constant to keep this phase velocity and average beam velocity identical as the beam energy was extracted. This enabled them to continue extracting beam energy even beyond the point at which the system normally saturates and synchrotron oscillations begin, and to achieve an energy extraction rate approximately four times higher than for an un-tapered dielectric.

Notice that tapering in Cerenkov sources is conceptually similar to tapering in conventional fast-wave free-electron lasers (FELs), in which an undulator's properties are varied along its length, generally to maintain the FEL resonance condition as the beam loses energy to the laser field. ${ }^{23}$ However, in both FELs and Cerenkov sources, the general rule that one should taper to maintain the resonant or synchronous condition may not optimize the overall system. Instead, attention must be paid to the nuances of electron motion in phase space, and to the details of the full system being optimized. In some cases, tapering in FELs does not improve system performance, as shown at the Jefferson Lab IR Demo FEL. ${ }^{24}$ In other cases an inverse taper — where the undulator properties are adjusted to increase the resonant energy while the beam energy decreases, thereby moving the system away from resonance - actually provides optimal system performance. ${ }^{25}$ And even where maintaining the resonant or synchronous condition is required to optimize system performance, opti- 
mized tapers in both $\mathrm{FELs}^{23}$ and Cerenkov sources ${ }^{14}$ may require an undulator magnetic field strength (for FELs) or permittivity (for Cerenkov sources) which has an oscillatory component rather than a smooth monotonic variation.

Previous studies of tapering in Cerenkov sources primarily used a combination of linear theory and macro-particle models. While both these models allow investigation of the small signal regime and the macro-particle model extends this into saturation, neither provides access to the nuances of electron motion in phase space during the beam-wave interaction, which are required for a fuller understanding and optimization of the interaction. Such granularity can only be provided by PIC codes.

In this paper, we consider linear permittivity tapers over the entire interaction region, as well as linear tapers starting where saturation would begin in the absence of tapering. This work builds on previous studies of an un-tapered device ${ }^{7}$ operating near $8 \mathrm{GHz}$. In that work, a radially profiled beam was studied, while in the present case an annular beam near the dielectric vacuum interface was used. The baseline case is analyzed using linear theory, a 2-D Particle-In-Cell (PIC) simulation, and a macro-particle model; the purpose of the macroparticle model is to bridge the gap between the linear theory and PIC simulations. Linear tapers over the entire interaction length increase efficiency, but not optimally due to detuning at the front end of the taper and failure to adequately track beam momentum through synchrotron oscillations. Some improvement is found using a taper only near saturation by improved tracking of beam momentum, and systematic study of linear tapers over various sections of the interaction length is developed using 2-D PIC simulations.

\section{BASELINE CASE: UN-TAPERED SOURCE}

\section{A. Linear Theory}

Figure 1 shows the configuration for the Cerenkov source, consisting of a perfectly conducting cylindrical waveguide of radius $R_{0}$ having a dielectric liner with inner radius $R_{d}$ located immediately inside the waveguide. An annular electron beam of uniform number density is located between radii $R_{b 1}$ and $R_{b 2}$. The beam has axial velocity $v_{0}=\beta c$, is assumed to be cold, and is guided by a large axial magnetic field so that the beam motion is longitudinal and cyclotron instabilities are unimportant. For the baseline case we assume 


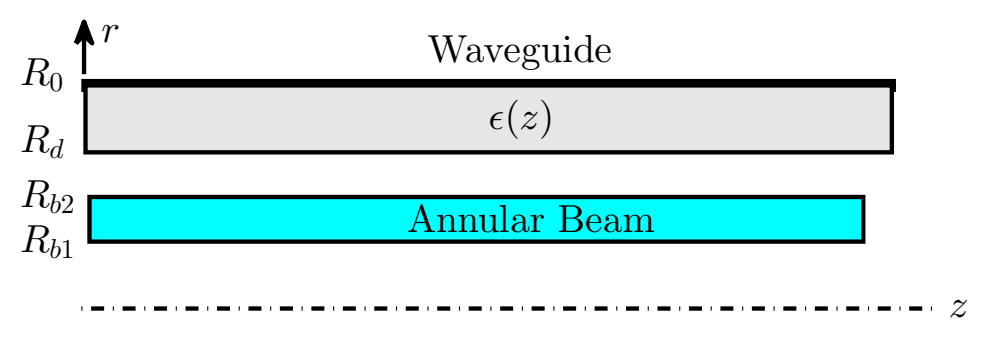

Annular Beam

$\epsilon(z)$

FIG. 1. Configuration of the Cerenkov source.

$\epsilon(z)=\epsilon$ is constant. Assuming a harmonic solution of the form $e^{i\left(k_{z} z-\omega t\right)}$, the defining equations for the axial electric field for $\mathrm{TM}_{0 n}$ modes are given by

$$
\begin{aligned}
{\left[\frac{1}{r} \frac{d}{d r}\left(r \frac{d}{d r}\right)-\kappa^{2}\right] E_{z}(r) } & =0, & 0 & \leq r \leq R_{b 1}, \\
{\left[\frac{1}{r} \frac{d}{d r}\left(r \frac{d}{d r}\right)-Q^{2}\right] E_{z}(r) } & =0, & R_{b 1} & \leq r \leq R_{b 2}, \\
{\left[\frac{1}{r} \frac{d}{d r}\left(r \frac{d}{d r}\right)-\kappa^{2}\right] E_{z}(r) } & =0, & R_{b 2} & \leq r \leq R_{d}, \\
{\left[\frac{1}{r} \frac{d}{d r}\left(r \frac{d}{d r}\right)+\kappa_{\epsilon}^{2}\right] E_{z}(r) } & =0, & R_{d} & \leq r \leq R_{0},
\end{aligned}
$$

where

$$
\begin{aligned}
\kappa^{2} & =k_{z}^{2}-\frac{\omega^{2}}{c^{2}}, \\
\kappa_{\epsilon}^{2} & =\frac{\epsilon \omega^{2}}{c^{2}}-k_{z}^{2}, \\
Q^{2} & =\kappa^{2}\left(1-\frac{\omega_{b}^{2}}{\Omega^{2}}\right) .
\end{aligned}
$$

The beam resonance term is given by

$$
\Omega^{2}=\gamma^{3}\left(\omega-k_{z} \beta c\right)^{2} .
$$

Here $\omega_{b}^{2}=q^{2} n_{0} / m \epsilon_{0}, \gamma=1 / \sqrt{1-\beta^{2}}$ is the relativistic factor, $q$ is the electron charge, $n_{0}$ is the beam number density, $m$ is the electron mass, $\epsilon_{0}$ is the permittivity of free space, $\epsilon$ is the relative permittivity of the dielectric sleeve, and $c$ is the speed of light. The solutions to Eqs. (1)-(4) are given by 


$$
\begin{array}{lrl}
E_{z}(r)=E_{1} I_{0}(\kappa r), & 0 \leq r \leq R_{b 1}, \\
E_{z}(r)=E_{2} I_{0}(Q r)+E_{3} K_{0}(Q r), & R_{b 1} \leq r \leq R_{b 2}, \\
E_{z}(r)=E_{4} I_{0}(\kappa r)+E_{5} K_{0}(\kappa r), & R_{b 2} \leq r \leq R_{d}, \\
E_{z}(r)=E_{6} J_{0}\left(\kappa_{\epsilon} r\right)+E_{7} Y_{0}\left(\kappa_{\epsilon} r\right), & R_{d} \leq r \leq R_{0} .
\end{array}
$$

Since we are considering only TM modes and there is no transverse beam motion due to the large axial magnetic field, the radial electric field for the vacuum and dielectric regions can be determined from

$$
\begin{array}{rlrl}
E_{r}=\frac{i k_{z} c^{2}}{\omega^{2}-k_{z}^{2} c^{2}} \frac{d E_{z}}{d r}, & & \leq r \leq R_{d} \\
E_{r}=\frac{i k_{z} c^{2}}{\epsilon \omega^{2}-k_{z}^{2} c^{2}} \frac{d E_{z}}{d r}, & R_{d} \leq r \leq R_{0}
\end{array}
$$

using the appropriate expression for the axial electric field in each of the four regions. Boundary conditions for the axial electric field and the radial electric displacement are then applied at the waveguide wall, the vacuum-beam interfaces, and the vacuum-dielectric interface to determine the dispersion relation, given by

$$
\operatorname{det}\left[\mathbf{D}\left(k_{z}, \omega\right)\right]=0
$$

where the elements of the matrix $\mathbf{D}$ are defined in the Appendix. Without the presence of the beam, the dispersion relation defined in Eq. (15) reduces to the familiar dispersion relation

$$
\frac{\kappa_{\epsilon}}{\epsilon \kappa} \frac{I_{1}\left(\kappa R_{d}\right)}{I_{0}\left(\kappa R_{d}\right)}+\frac{J_{0}\left(\kappa_{\epsilon} R_{0}\right) Y_{1}\left(\kappa_{\epsilon} R_{d}\right)-J_{1}\left(\kappa_{\epsilon} R_{d}\right) Y_{0}\left(\kappa_{\epsilon} R_{0}\right)}{J_{0}\left(\kappa_{\epsilon} R_{d}\right) Y_{0}\left(\kappa_{\epsilon} R_{0}\right)-J_{0}\left(\kappa_{\epsilon} R_{0}\right) Y_{0}\left(\kappa_{\epsilon} R_{d}\right)}=0
$$

\section{B. Macro-Particle Model}

Unlike the linear theory discussed above, a macro-particle model of the Cerenkov source can be used to provide information about the saturation of the instability and the transition from the linear to the saturated regime. An extensive examination of this particular geometry $^{6,7}$ was previously analyzed using such a model. The electromagnetic field in the waveguide is expressed in terms of the normal modes of the system without the presence of 
the electron beam, in this case the $\mathrm{TM}_{01}$ mode. The axial electric field component can be written as

$$
\begin{array}{lr}
E_{z}=E(z) I_{0}(\kappa r) \cos \Psi(z), & 0 \leq r \leq R_{d}, \\
E_{z}=E(z)\left[a J_{0}\left(\kappa_{\epsilon} r\right)+b Y_{0}\left(\kappa_{\epsilon} r\right)\right] \cos \Psi(z), & R_{d} \leq r \leq R_{0}
\end{array}
$$

where

$$
\Psi(z)=\int_{0}^{z} k_{z}\left(z^{\prime}\right) d z^{\prime}-\omega t
$$

and the local field amplitudes and local wave numbers are assumed to be slowly varying functions of $z$,

$$
\left|\frac{1}{E} \frac{\partial E}{\partial z}\right| \ll k_{z} \quad \text { and } \quad\left|\frac{1}{k_{z}} \frac{\partial k_{z}}{\partial z}\right| \ll k_{z} .
$$

The constant $a$ and $b$ are determined from the boundary conditions and are given by

$$
a=\left(\frac{\pi}{2}\right) \kappa_{\epsilon} R_{d}\left[\left(\frac{\kappa_{\epsilon}}{\epsilon \kappa}\right) I_{1}\left(\kappa R_{d}\right) Y_{0}\left(\kappa_{\epsilon} R_{d}\right)-I_{0}\left(\kappa R_{d}\right) Y_{1}\left(\kappa_{\epsilon} R_{d}\right)\right]
$$

and

$$
b=-\left(\frac{\pi}{2}\right) \kappa_{\epsilon} R_{d}\left[\left(\frac{\kappa_{\epsilon}}{\epsilon \kappa}\right) I_{1}\left(\kappa R_{d}\right) J_{0}\left(\kappa_{\epsilon} R_{d}\right)-I_{0}\left(\kappa R_{d}\right) J_{1}\left(\kappa_{\epsilon} R_{d}\right)\right]
$$

Following the procedure previously shown, ${ }^{6,7}$ it is possible to write the following equations describing the evolution of the amplitude and phase of the electromagnetic wave in terms of the beam current:

$$
\begin{aligned}
2 k_{z} \frac{d E}{d z} & =\frac{2 \mu_{0} c^{2} \kappa^{2}}{\pi A R_{d}^{2}} \int_{0}^{\frac{2 \pi}{\omega}} d t \int_{0}^{R_{d}} r J_{z} I_{0}(\kappa r) \cos \Psi d r \\
\left(\frac{\omega^{2}}{c^{2}}-\kappa^{2}-k_{z}^{2}\right) E & =\frac{2 \mu_{0} c^{2} \kappa^{2}}{\pi A R_{d}^{2}} \int_{0}^{\frac{2 \pi}{\omega}} d t \int_{0}^{R_{d}} r J_{z} I_{0}(\kappa r) \sin \Psi d r
\end{aligned}
$$

where the constant $A$ is given by

$$
\begin{array}{r}
A=\left(\frac{\epsilon-1}{\epsilon}\right)\left(I_{1}^{2}\left(\kappa R_{d}\right)-\frac{\epsilon k_{z 0}^{2}}{\kappa_{\epsilon}^{2}} I_{0}^{2}\left(\kappa R_{d}\right)+\frac{2}{\kappa R_{d}} \frac{\epsilon \omega^{2}}{c^{2} \kappa_{\epsilon}^{2}} I_{0}\left(\kappa R_{d}\right) I_{1}\left(\kappa R_{d}\right)\right) \\
+\epsilon \frac{R_{0}^{2}}{R_{d}^{2}} \frac{\kappa^{2}}{\kappa_{\epsilon}^{2}}\left[a J_{1}\left(\kappa_{\epsilon} R_{0}\right)+b Y_{1}\left(\kappa_{\epsilon} R_{0}\right)\right]^{2}
\end{array}
$$

the variation in axial wavenumber is represented in terms of a relative phase $\psi(z)$

$$
k_{z}(z)=k_{z 0}+\frac{d \psi}{d z}
$$


and $k_{z 0}$ is the initial wavenumber determined from the cold dispersion relation. The macroscopic current is found by summing over the individual trajectories of an ensemble of individual macro particles,

$$
J_{z}\left(z, \tau_{p q}\right)=-e n_{b}\left(r_{q}\right) \frac{2 \pi v_{0}}{N_{\tau}} \sum_{p=1}^{N_{\tau}} \delta\left(\tau-\tau_{p q}(z)\right),
$$

where $\tau=\omega t, N_{\tau}$ is the number of macro particles distributed over $2 \pi$ in phase, and $N_{r}$ is the number of macro particles in radius. Here the indices $p$ and $q$ refer to the macro particle's position in radius or phase respectively. The relativistic dynamical equations can be written for the $(p q)^{\text {th }}$ macro particle as

$$
\frac{d \tau_{p q}}{d z}=\frac{\omega}{c \beta_{p q}}=\frac{k_{0}}{\beta_{p q}}
$$

and

$$
\frac{d \beta_{p q}}{d z}=-\frac{e}{m c^{2}}\left[\frac{\left(1-\beta_{p q}\right)^{3 / 2}}{\beta_{p q}}\right] E(z) J_{0}\left(\kappa r_{q}\right) \cos \left(k_{z 0} z+\frac{d \psi}{d z} z-\tau_{p q}\right) .
$$

Equations (23)-(29) constitute $2 N_{r} N_{\tau}+2$ differential equations $\left(N_{r} N_{\tau}\right.$ equations for particle phase, $N_{r} N_{\tau}$ equations for particle velocity, and 2 equations for the wave amplitude and phase) which can be solved numerically.

\section{Numerical Results for Baseline Case}

For numerical calculations of the baseline case we assume a waveguide with: $R_{0}=2 \mathrm{~cm}$, $R_{d}=1.5 \mathrm{~cm}$, and $\epsilon=4$. Figure 2 shows the cold dispersion relation from Eq. (16) for the $\mathrm{TM}_{01}$ mode, along with the beam mode for an electron beam with a nominal injection energy of $340 \mathrm{keV}$; their intersection indicates an operating frequency of $8.12 \mathrm{GHz}$. The full dispersion relation allows for solutions with complex $k_{z}$ when driven by an electron beam. Figure 3 shows the solution of Eq. (15) for the system when driven by a $340 \mathrm{keV}, 50 \mathrm{~A}$ annular electron beam with $R_{b 1}=1.25 \mathrm{~cm}$ and $R_{b 2}=1.45 \mathrm{~cm}$. A maximum spatial growth rate, $\Gamma=-\operatorname{Im}\left(k_{z}\right)=8.52 \mathrm{~m}^{-1}$, occurs at $8.12 \mathrm{GHz}$, with $\operatorname{Re}\left(k_{z}\right)=217.6 \mathrm{~m}^{-1}$.

The macro-particle model and PIC simulations are required to determine the performance of the amplifier near nonlinear saturation. The geometry shown in Fig. 1 was simulated with OOPIC ${ }^{26}$ in $r-z$ geometry with mesh cell sizes of $\Delta r=\Delta z=0.5 \mathrm{~mm}$ and a time step of $\Delta t=1.0 \mathrm{ps}$. The overall axial length of the simulation was 1.5 meters, giving $1.2 \times 10^{5}$ mesh cells with a maximum of $\sim 8 \times 10^{5}$ macro particles. Figure 4 shows the injected current 


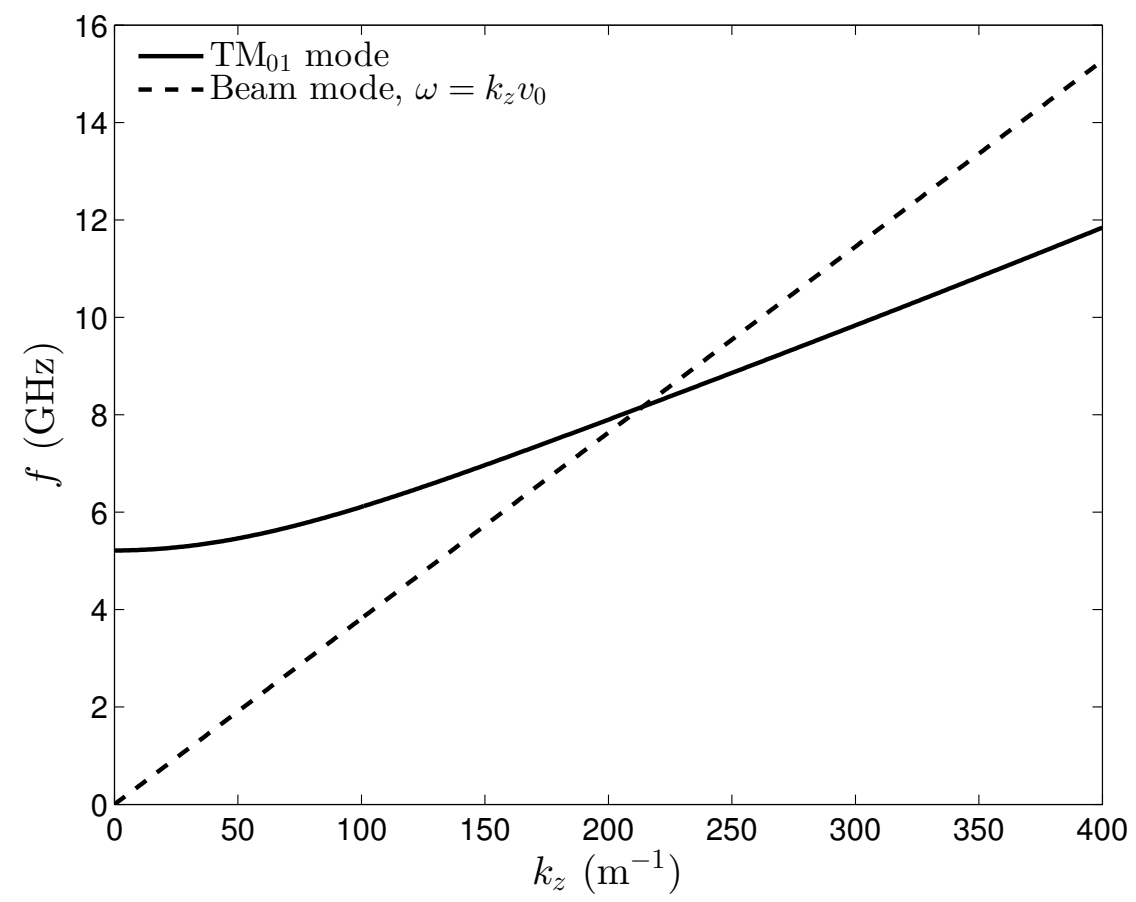

FIG. 2. Cold dispersion relation showing nominal $340 \mathrm{keV}$ beam mode.
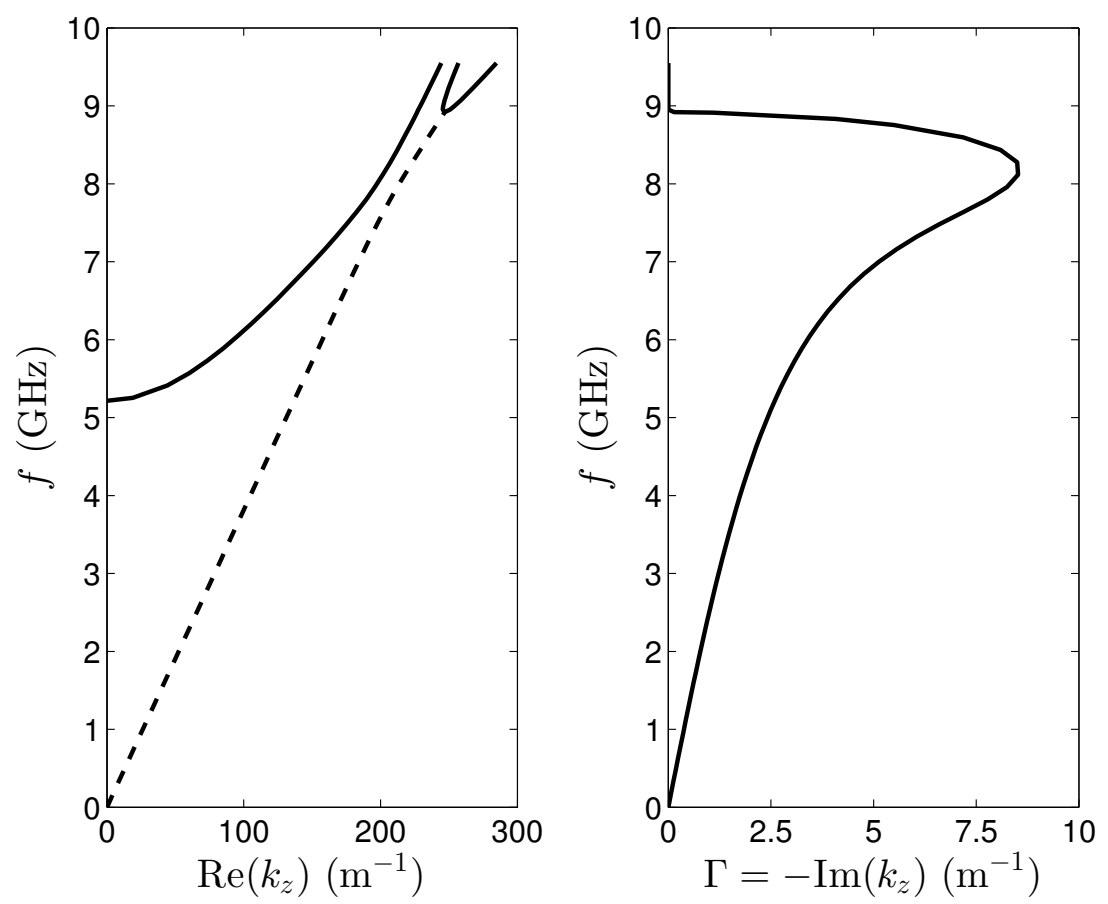

FIG. 3. Real and imaginary solution of the dispersion relation. The dashed line represents the complex conjugate solution pair. 


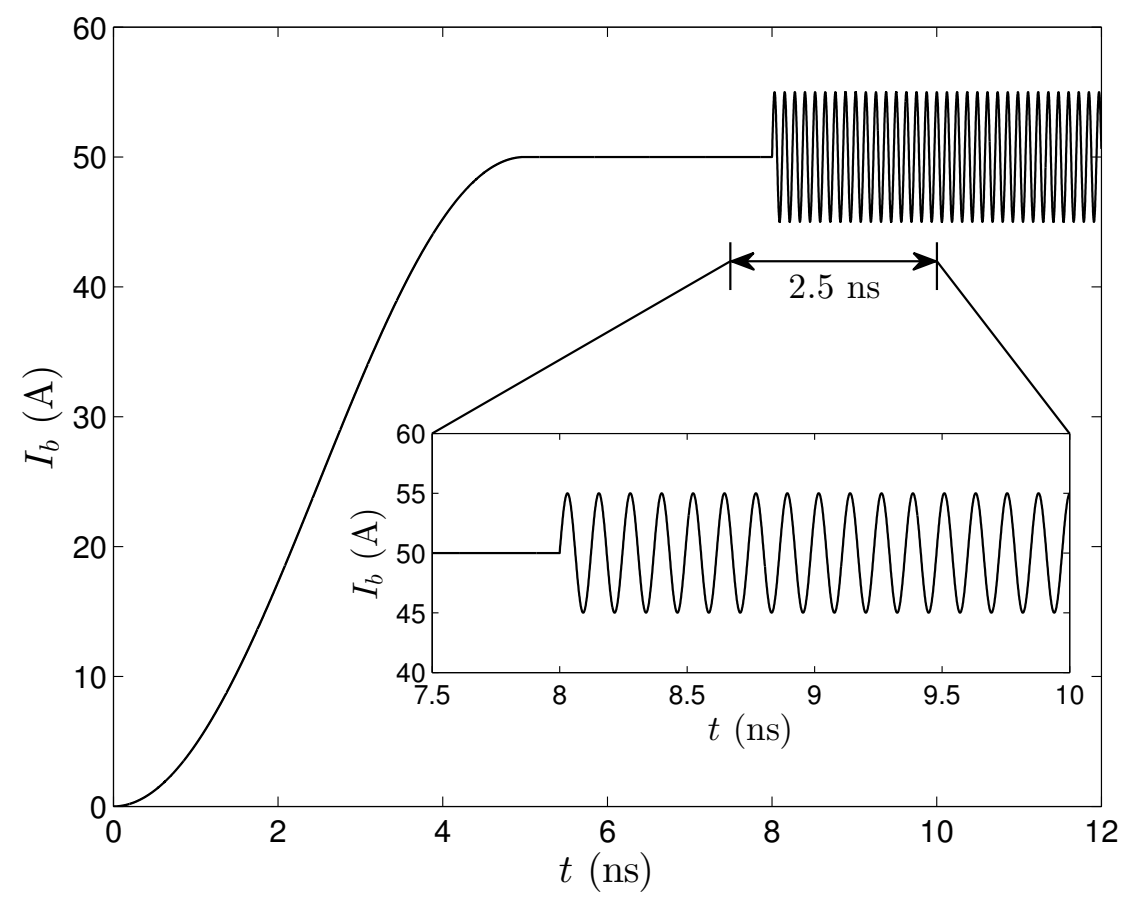

FIG. 4. OOPIC current pulse showing the temporal isolation of the beam head and harmonic density perturbation.

pulse, which contains a $10 \%$ sinusoidal density perturbation at $8.12 \mathrm{GHz}$ to serve as the seed signal. To prevent coherent spontaneous emission (CSE) ${ }^{7}$ associated with the beam head, the current is slowly ramped up to the baseline value of $50 \mathrm{~A}$ over a period of $5 \mathrm{~ns}$, and the seed signal is turned on 3 ns later. This method also provides a degree of space and time isolation of the transient and steady state signals. Numerical simulations for the Cerenkov source were also solved using the macro-particle model for the $\mathrm{TM}_{01}$ mode for our set of parameters using $N_{r}=4$ and $N_{\tau}=128$. The particles entering the amplifier were uniformly distributed in phase, and the initial axial electric field on axis was set to $25 \mathrm{kV} / \mathrm{m}$, to be consistent with the PIC simulations.

Figure 5 shows results for $E_{z}(z)$, along the waveguide axis from OOPIC simulations along with the envelope of $E_{z}(z)$ from the macro-particle model. The spatial growth rates are determined to be $8.04 \mathrm{~m}^{-1}$ and $8.39 \mathrm{~m}^{-1}$ for the OOPIC and macro-particle model respectively, compared with the linear theory prediction of $8.52 \mathrm{~m}^{-1}$. The mode structure for the axial electric field at $z=0.3 \mathrm{~m}$ found by OOPIC is in excellent agreement with that calculated from the linear theory as shown in Fig. 6. Figure 7 shows the evolution 
of the microwave power and average beam energy from the OOPIC simulations and the macro-particle model. OOPIC and the macro-particle model show saturation occurring at $z=0.55 \mathrm{~m}$ at powers of $3.2 \mathrm{MW}$ and $3.04 \mathrm{MW}$, and with a corresponding average beam energy at saturation of $275 \mathrm{keV}$ and $279 \mathrm{keV}$, respectively. These values correspond to efficiencies of $18.8 \%$ and $17.9 \%$ for the OOPIC and macro-particle simulations respectively. Figure 8 shows the longitudinal phase space of the beam found in OOPIC, clearly showing the beam starting to execute synchrotron oscillations near $z=0.55 \mathrm{~m}$. Maximum beam energy loss occurs at the same location with the beam regaining a maximum amount of energy at $z=0.76 \mathrm{~m}$, where the microwave output power is at a minimum. Energy distributions for the beam at several locations along the amplifier were also extracted from the PIC data and are shown in Fig. 9. Prior to saturation the particle energies are distributed around the $340 \mathrm{keV}$ injection energy with a mean value of $325 \mathrm{keV}$. As the wave starts to saturate at $z=0.55 \mathrm{~m}$ the particles become trapped at approximately $250 \mathrm{keV}$ with a mean value of $275 \mathrm{keV}$. The mean energy is somewhat larger than the trapped energy due to the high energy tail in the distribution located above $250 \mathrm{keV}$. At $z=0.76 \mathrm{~m}$ the wave has given energy back to the particles with the bulk of the particles distributed near $350 \mathrm{keV}$ with a mean value of $326 \mathrm{keV}$. Note that the energy spread, or longitudinal emittance, has been increased by this process. The dynamics of the energy exchange between the wave and the particles are shown more clearly in Fig. 10, which shows the axial electric field, beam current, and axial wave number, along $z$. The bunch locations are indicated by the fiducial markers on the axial electric field plot. The bunch starts in a decelerating phase of the wave for $z<0.55 \mathrm{~m}$; between $z=0.55 \mathrm{~m}$ and $z=0.76 \mathrm{~m}$ the bunch is in an accelerating phase of the wave where the wave energy is decreasing. After $z=0.76 \mathrm{~m}$ the bunch again enters a decelerating phase of the wave and the microwave power starts to increase again. The instantaneous wave amplitude and phase were determined by evaluating the Hilbert transform of the axial electric field, $E_{z}\left(z ; t_{0}\right)$ with $t_{0}$ sufficiently large that the steady state regime has been established. The axial wave number associated with the interaction is fairly constant with a value of $218 \mathrm{~m}^{-1}$ throughout the course of the interaction length. 


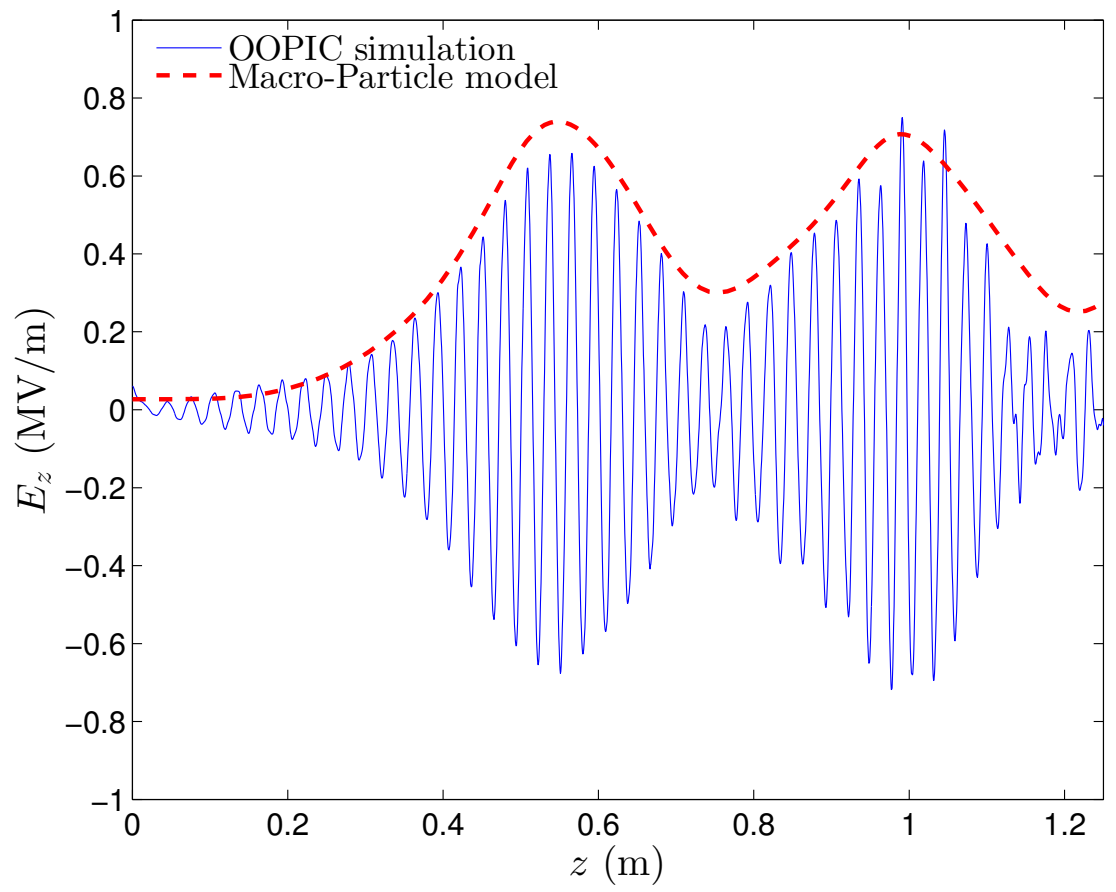

FIG. 5. Axial electric field on axis from OOPIC. The envelope of the electric field is shown for the macro-particle simulations.

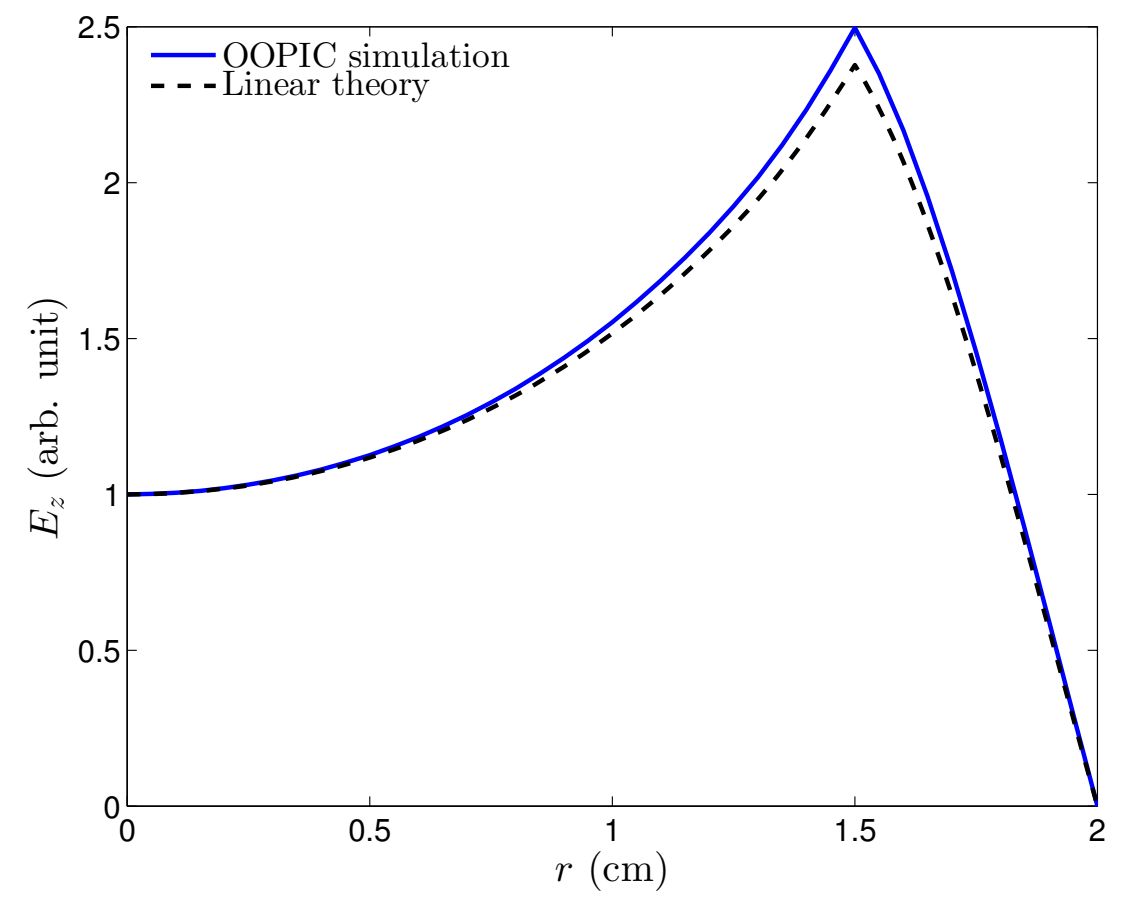

FIG. 6. Mode structure at $z=0.3 \mathrm{~m}$ for $\mathrm{TM}_{01}$ mode at $8.12 \mathrm{GHz}$. 

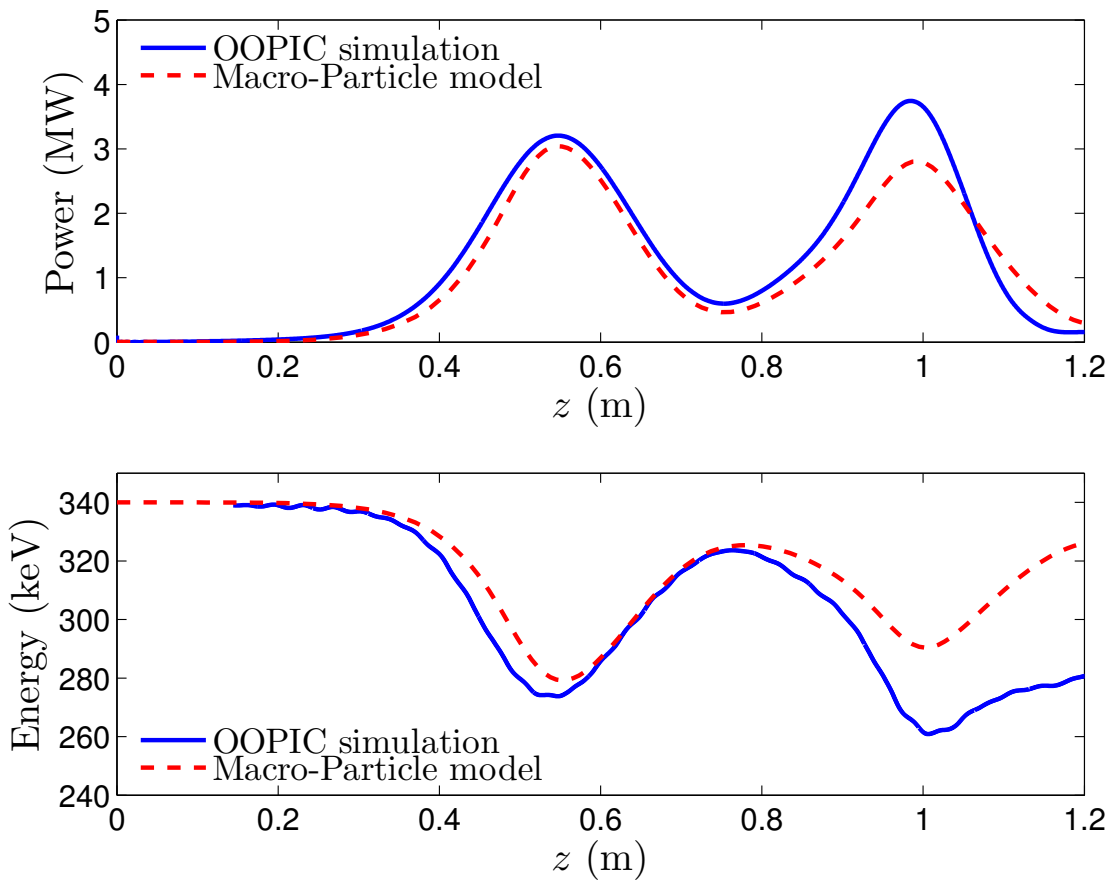

FIG. 7. $\mathrm{TM}_{01}$ power and beam energy loss.

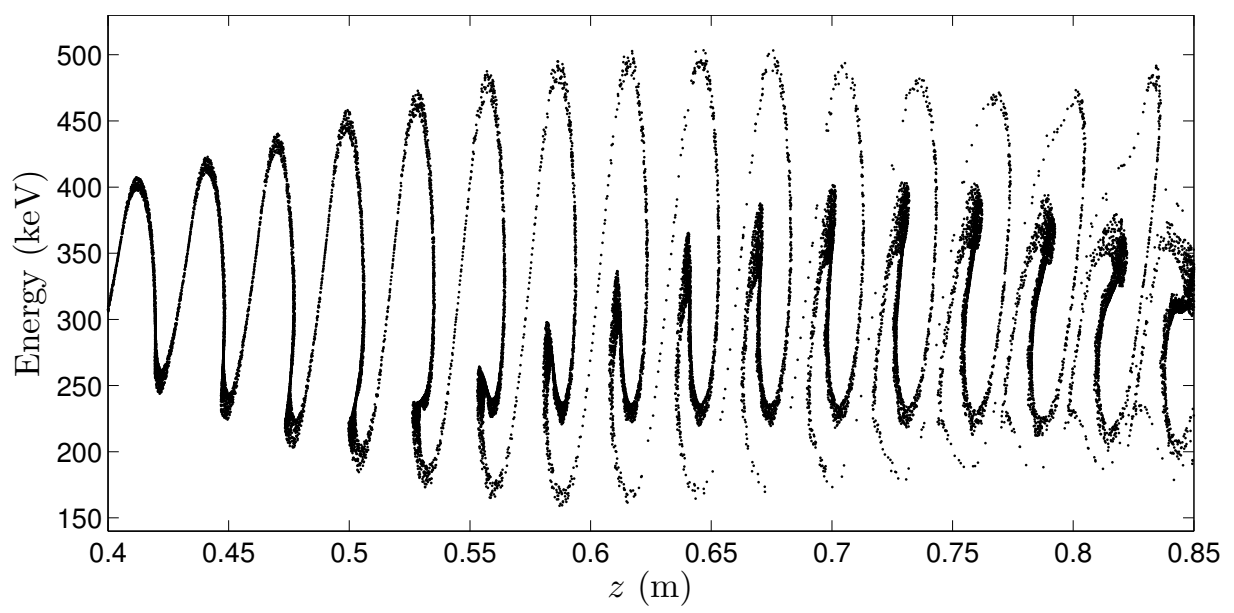

FIG. 8. Longitudinal phase space showing onset of beam trapping and synchrotron oscillations. 

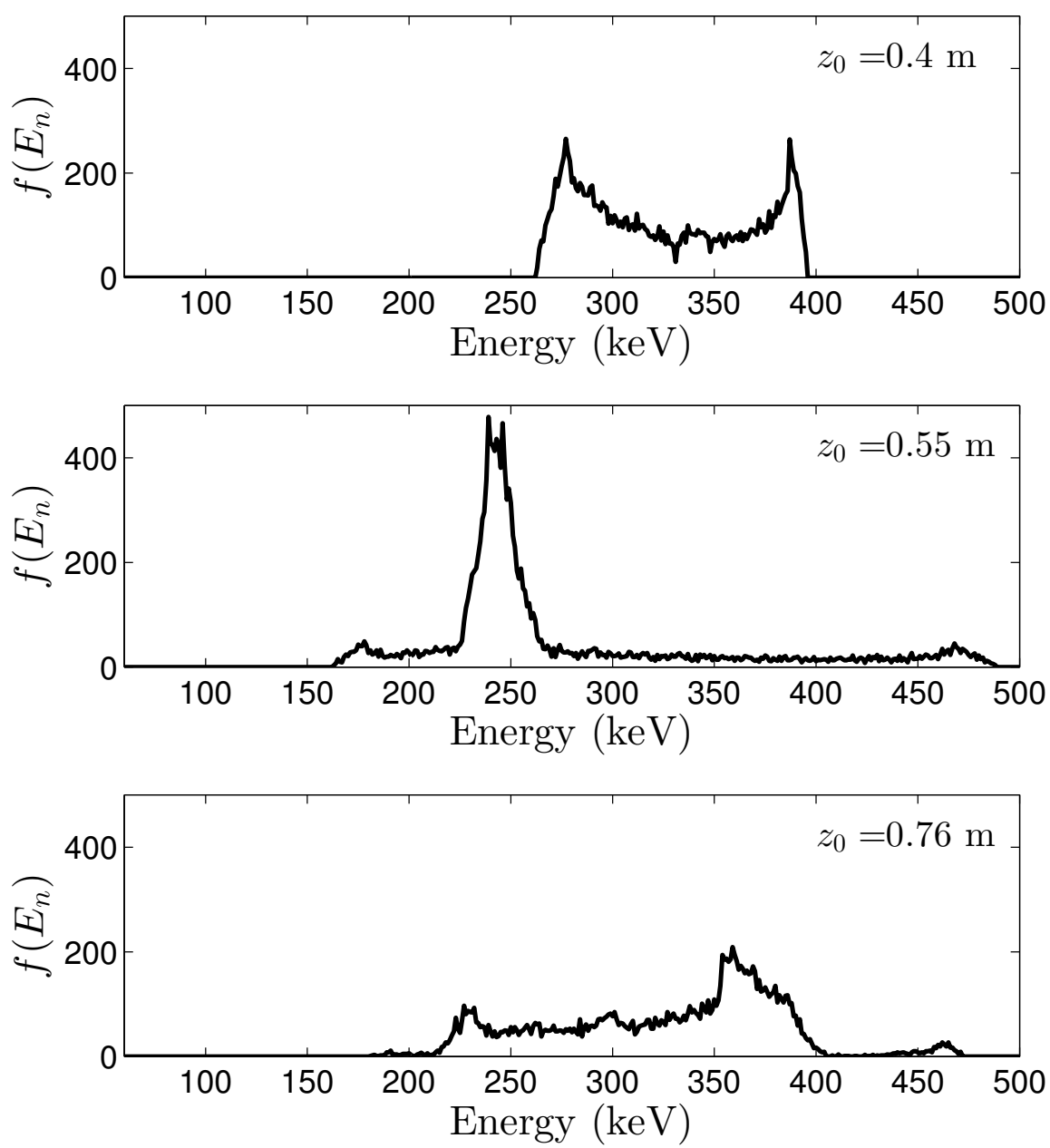

FIG. 9. Evolution of energy distribution. The bin sizes in energy are $1 \mathrm{keV}$. The thickness of the beam slice for sampled particles in the energy distribution was $2 \pi / k_{z}\left(z_{0}\right)$ centered at $z_{0}$. 

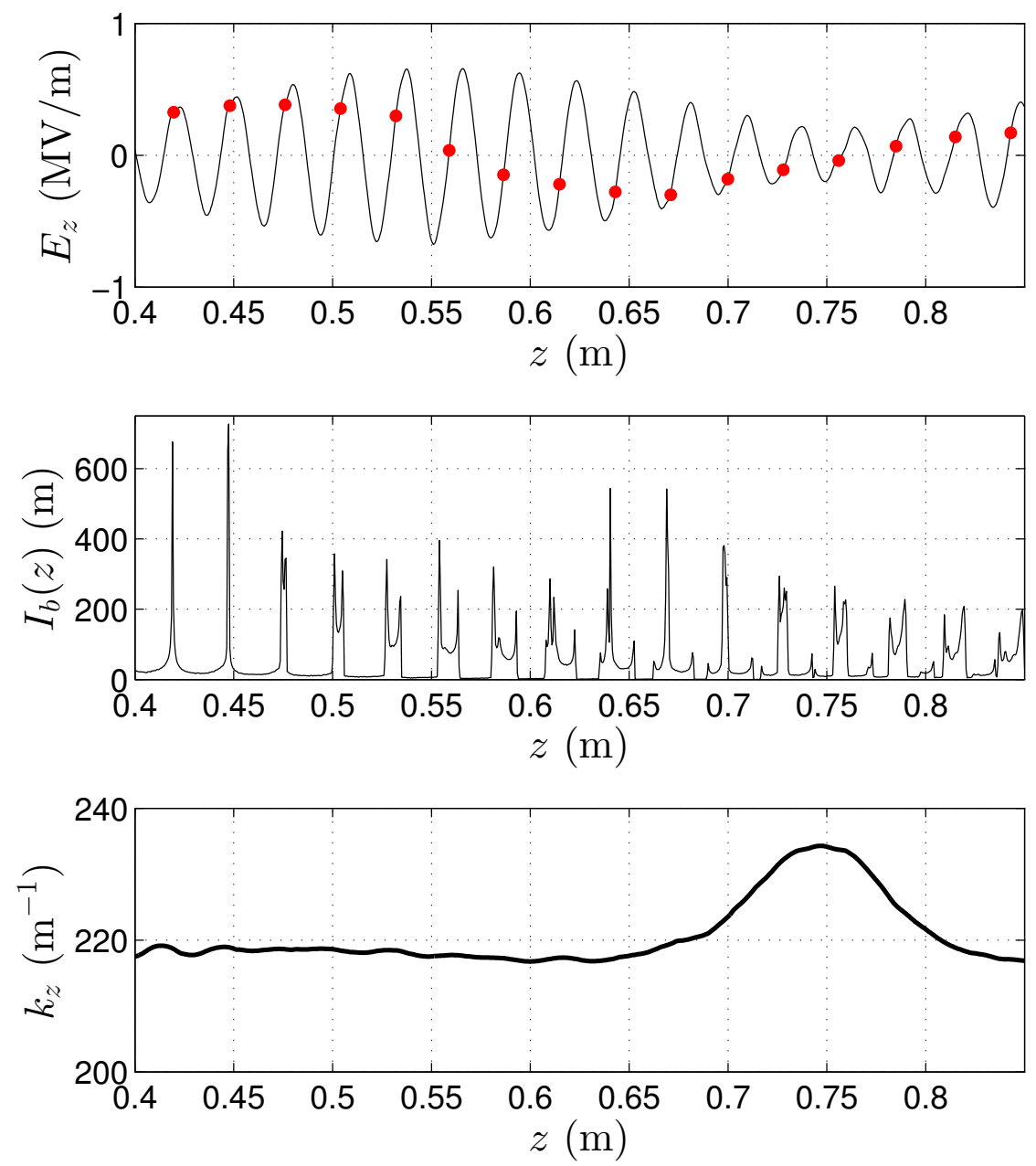

FIG. 10. Axial electric field, beam bunching showing relative phasing between field and bunches and evolution of axial wave number, $k_{z}$. 


\section{TAPERED SOURCE}

\section{A. Overview}

Properly tapering the dispersion properties of the slow wave structure along the length of the amplifier allows the phase velocity of the mode to better track the electron beam velocity as the beam gives up energy to the electromagnetic wave. This is illustrated conceptually in Fig. 11, which shows cold dispersion curves for several values of dielectric permittivity and several beam energies chosen to maintain synchronism at the operating frequency for our geometry. The objective is to choose the variation of dielectric permittivity with distance along the amplifier in such a way that the power in the electromagnetic wave is maximized. While simple in concept, this is more complicated in practice due to the dependence of the growth rate, modal structure,and dispersion properties on the dielectric permittivity and the changing beam energy. Figures 12 and 13 show plots of the variation of maximum growth rate and wave number with beam energy at the $8.12 \mathrm{GHz}$ operating frequency for several

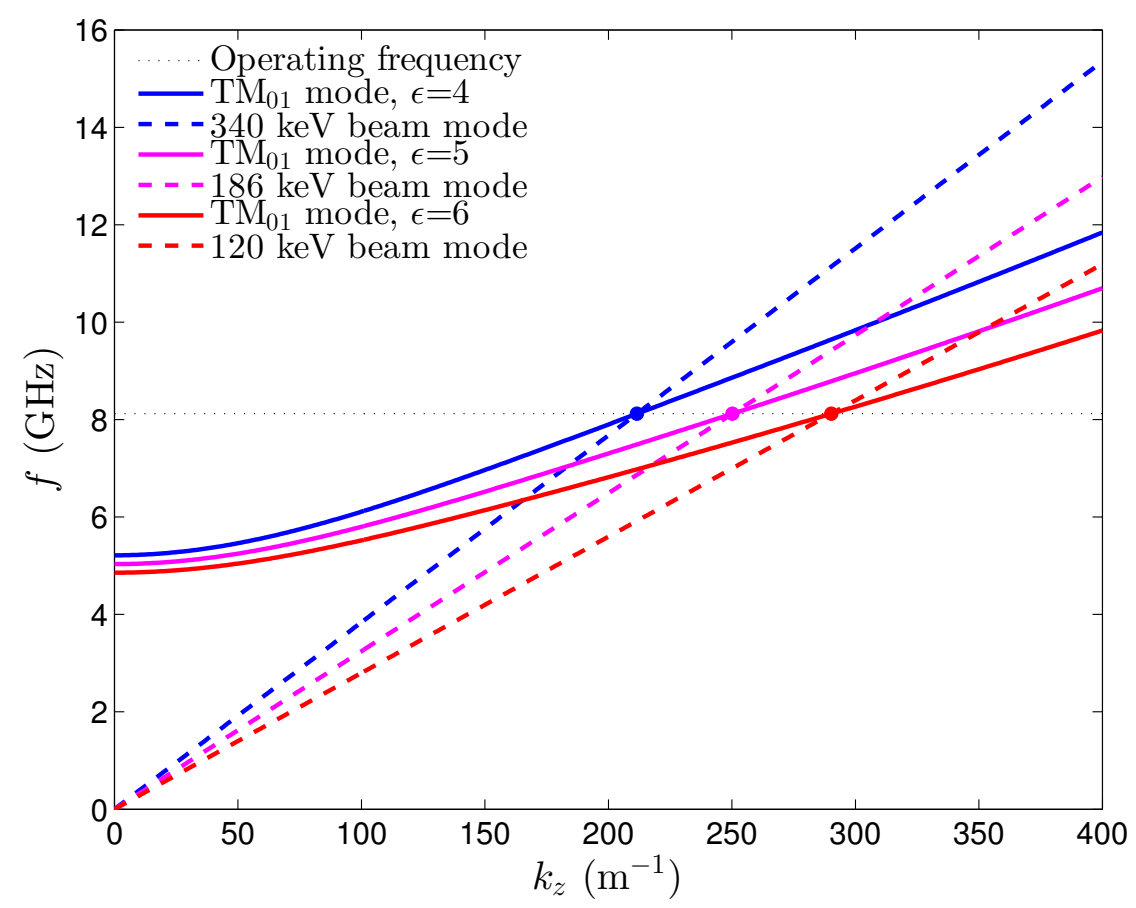

FIG. 11. TM $\mathrm{TM}_{01}$ dispersion curves for several values of dielectric permittivity and the associated synchronous beam modes for $8.12 \mathrm{GHz}$. 


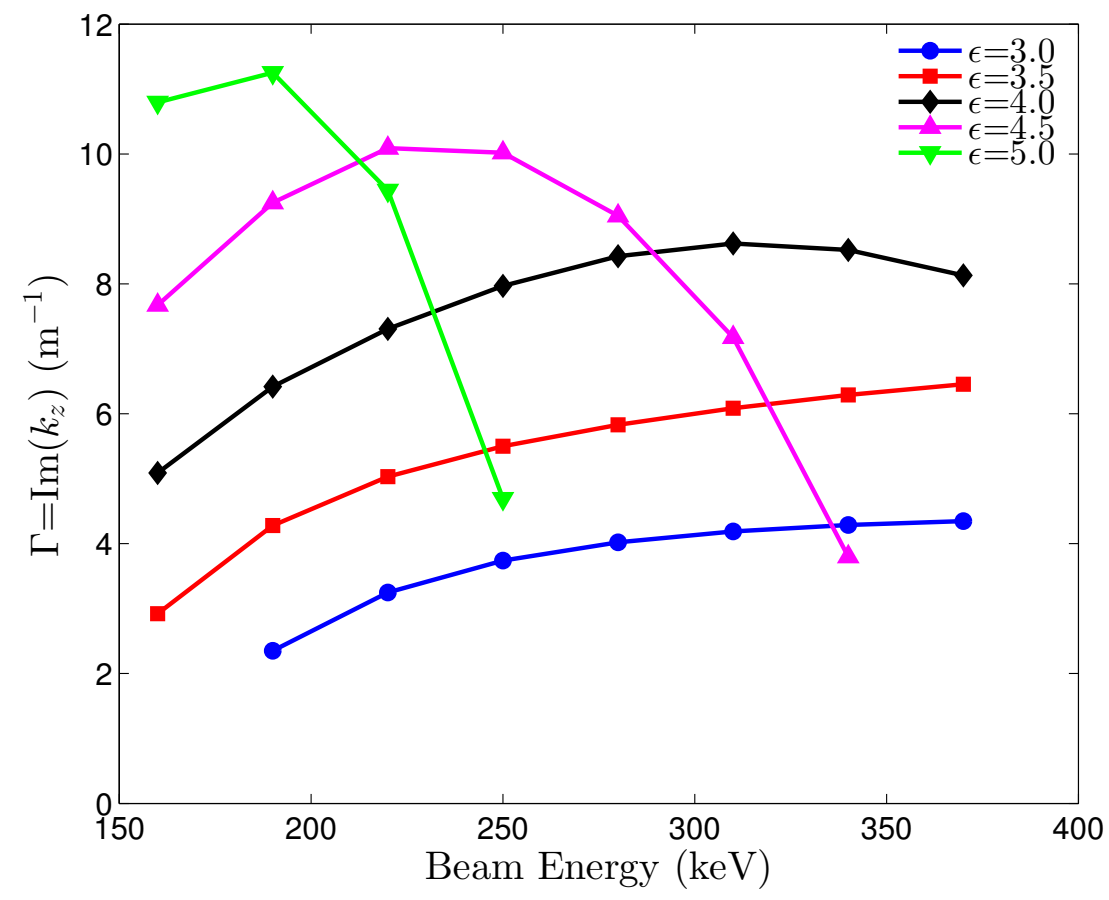

FIG. 12. Variation of growth rate with beam energy for spatially growing mode at $f=8.12 \mathrm{GHz}$.

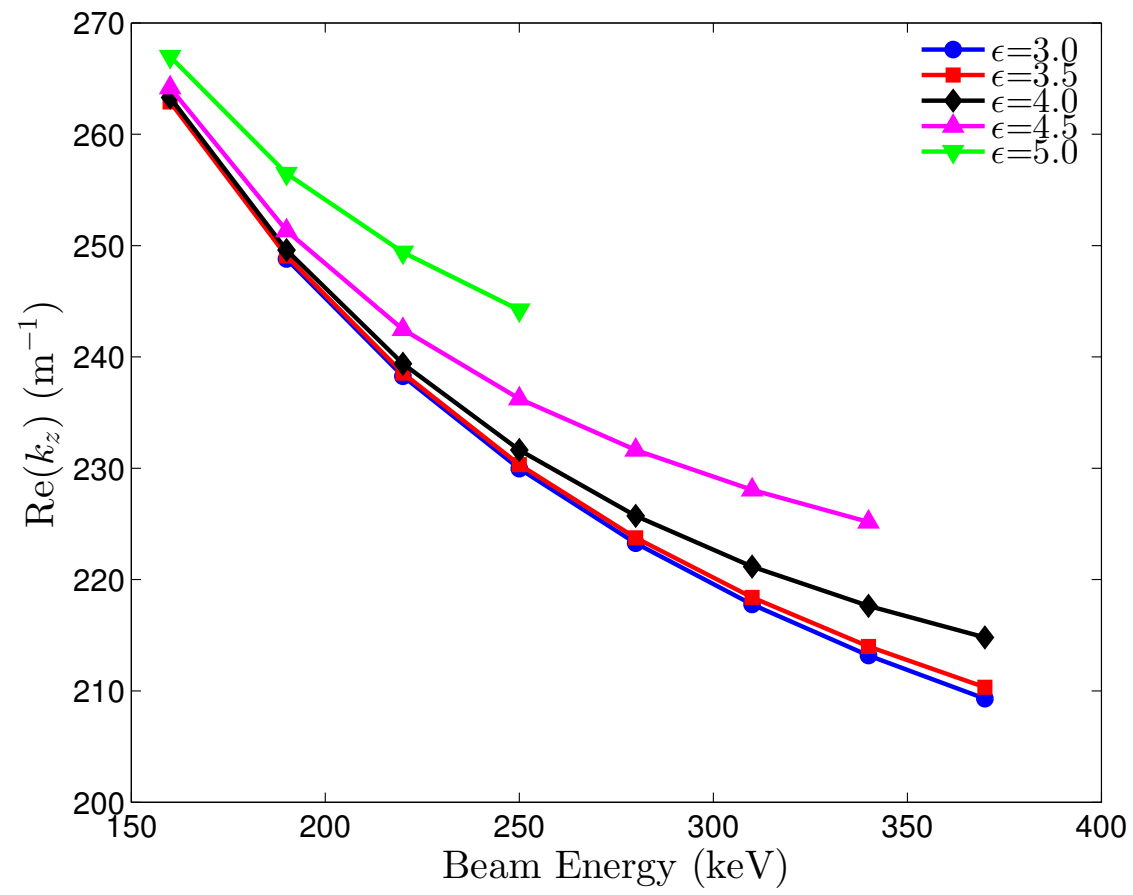

FIG. 13. Variation of wavenumber with beam energy for spatially growing mode at $f=8.12 \mathrm{GHz}$. 
values of dielectric permittivity respectively as calculated from Eq. (15). Also evident from Fig. 13 is that at lower beam energies, $k_{z}$ increases since the mode's phase speed, $\omega / k_{z}$ is decreasing, and therefore, $\kappa=\sqrt{k_{z}^{2}-(\omega / c)^{2}}$ will increase changing the modal structure within the beam region. To further complicate matters, Figs. 11-13 assume a mono-energetic beam, while the results of Fig. 9 show that the beam energy spread is significant, end evolves as the beam interacts with the wave.

In the next sections of this paper we will examine linear tapers to determine their effectiveness in enhancing the output power of the Cerenkov source. Figure 14 shows the two linear tapers under consideration in this paper. In the first, denoted as the "long" taper, the dielectric constant is varied linearly over the entire length of the source. The defining equations for $\epsilon(z)$ for the "long" taper are given by

$$
\epsilon(z)=\epsilon_{i}+\frac{d \epsilon}{d z} z
$$

where

$$
\frac{d \epsilon}{d z}=\frac{\epsilon_{f}-\epsilon_{i}}{L}
$$

In the second taper, denoted as the "short" taper, the dielectric constant remains at $\epsilon_{i}$ for $z \leq L_{i}$, and $\epsilon_{f}$ for $Z \geq L_{f}$. In the taper region, $L_{i} \leq z \leq L_{f}, \epsilon(z)$ is given by

$$
\epsilon(z)=\epsilon_{i}+\frac{d \epsilon}{d z}\left(z-L_{i}\right), \quad L_{i} \leq z \leq L_{f}
$$

where

$$
\frac{d \epsilon}{d z}=\frac{\epsilon_{f}-\epsilon_{i}}{L_{f}-L_{i}}
$$




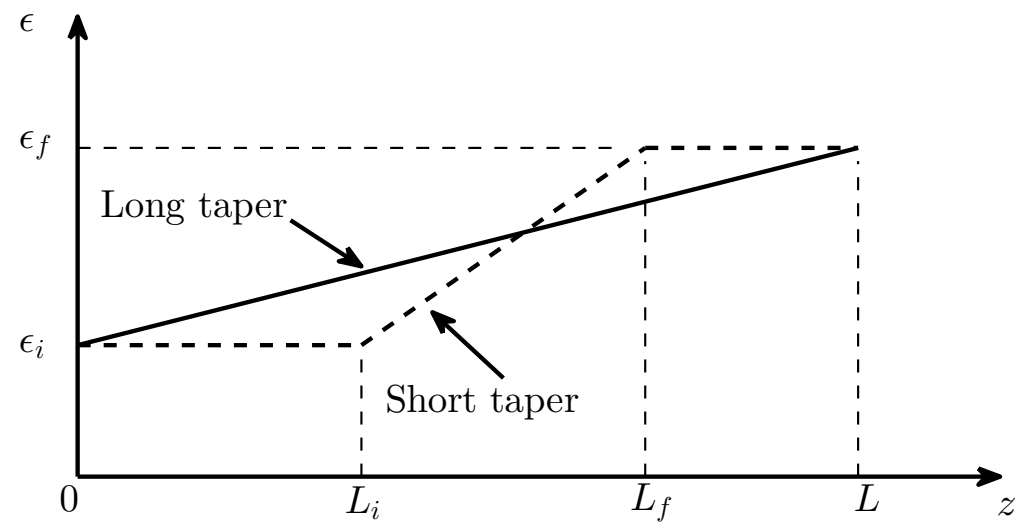

FIG. 14. Long and short linear taper configurations. 


\section{B. Linear Tapers Along the Entire Interaction Region ("Long" taper)}

First, we consider linear tapers extending across the entire $L=1.5 \mathrm{~m}$ interaction region from the point of beam injection to the end of the simulation volume. The initial value of dielectric constant at $z=0$ is $\epsilon_{i}=4$, corresponding to the baseline case, and simulations are run for final values of dielectric constant, $\left(\epsilon_{f}\right)$, ranging from 3 to 7 . The tapered dielectric was simulated in OOPIC using discrete annular disks of dielectric material $1.5 \mathrm{~cm}(30 \Delta z)$ thick along the length of the amplifier. Figure 15 summarizes the results of the "long" taper simulations, showing the maximum power output at the first and second synchrotron oscillation peaks as a function of taper. Figure 16 shows the evolution of the microwave power and average beam energy for the best performing taper $\left(\epsilon_{f}=5.8\right)$ compared with the untapered amplifier. The power at saturation for the tapered amplifier is $6.8 \mathrm{MW}$ with a beam energy at saturation of approximately $198 \mathrm{keV}$. This corresponds to an amplifier efficiency of approximately $40 \%$ compared to an efficiency of $18.8 \%$ for the un-tapered amplifier. The longitudinal phase space for $\epsilon_{f}=5.8$ is shown in Fig. 17. Saturation is delayed to about $z=0.6 \mathrm{~m}$ allowing continued growth of the wave before the onset of synchrotron oscillations. Note the more complicated structure seen after $z=0.65 \mathrm{~m}$ in this figure compared to the un-tapered data shown in Fig. 8, indicating the trapping of additioanl particles near $350 \mathrm{keV}$ not seen in the un-tapered case. Fig. 18 shows that at saturation the particles are distributed near $110 \mathrm{keV}$ compared to $250 \mathrm{keV}$ for the un-tapered source. Again, the presence of a high energy tail in the energy distribution skews the mean energy upward to about $198 \mathrm{keV}$, but the overall energy spread is similar in the tapered and un-tapered cases. At approximately $z=0.76 \mathrm{~m}$ the beam again starts to give energy to the wave. However, the peak output power at the second synchrotron oscillation peak is substantially lower than the first peak due to detuning of the instability, since the beam and wave are falling out of synchronism due to the changing dielectric constant in the taper. Figure 19 shows the relative phasing of the wave and the bunch, again with the bunch starting in a decelerating phase of the wave until approximately $z=0.6 \mathrm{~m}$ where it slips into an accelerating phase and starts to regain energy at the expense of the electromagnetic wave. It remains in the accelerating phase until approximately $z=0.76 \mathrm{~m}$ where the bunch again slips into a decelerating phase and the microwave field again starts to increase. By comparison with the un-tapered case shown in Fig. 10, the taper causes the bunch to stay in a decelerating phase for longer, and 
to be more quickly brought out of the accelerating phase once it enters it. The axial wave number, $k_{z}$ is increasing along the amplifier due to the tapering of the dielectric constant. The increasing value of $k_{z}$ along the amplifier allows the bunch to remain in an accelerating phase of the wave for a longer distance allowing for a larger microwave output power. An increase in the strength of the taper near saturation to decrease the phase velocity of the wave further to match the mean bunch velocity as the bunch enters synchrotron oscillations would potentially allow for improved energy extraction. In addition, tapering near the amplifier input does not significantly allow for improved performance, and may in fact degrade operation, due to premature detuning of the instability, when there is little change in mean beam energy. More will be discussed about this in the next section.

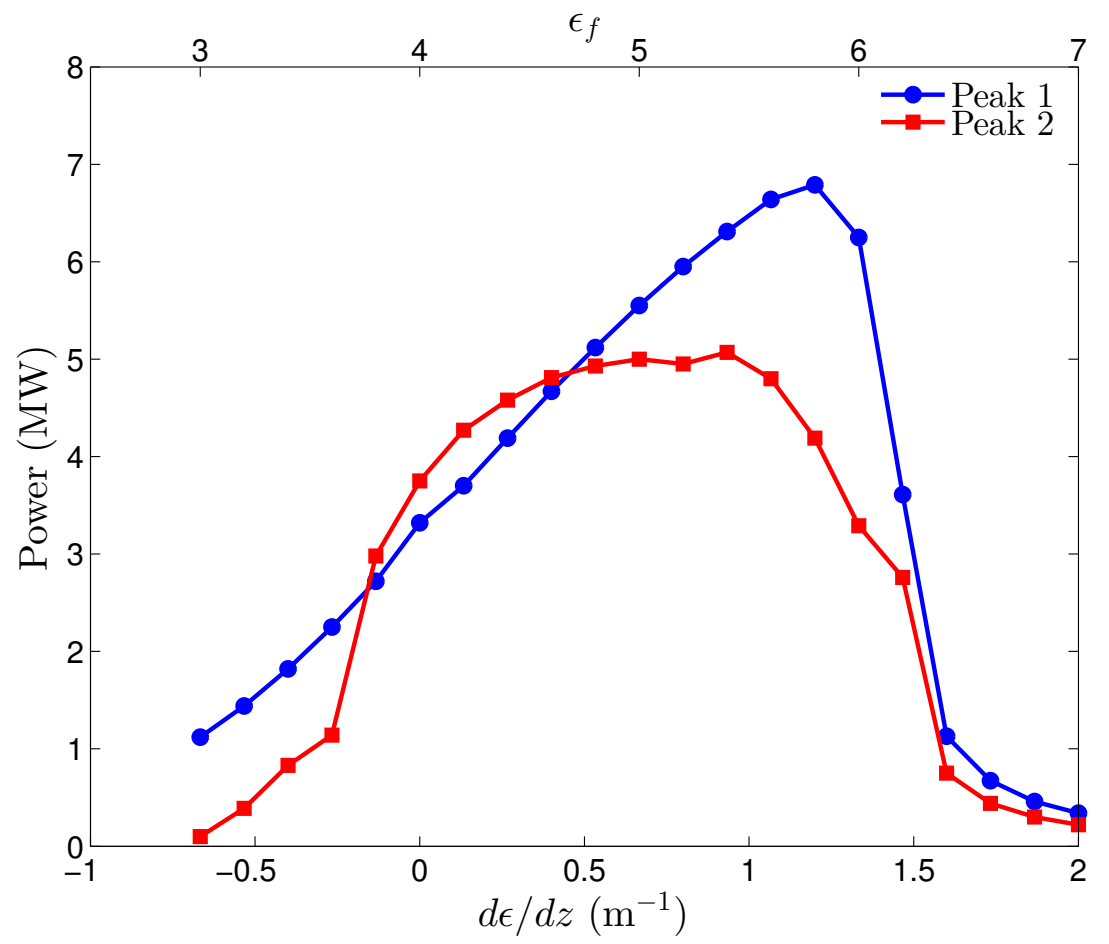

FIG. 15. Power output at first and second synchrotron oscillation peak for a Cerenkov amplifier with a linear taper extending over the entire interaction length. 

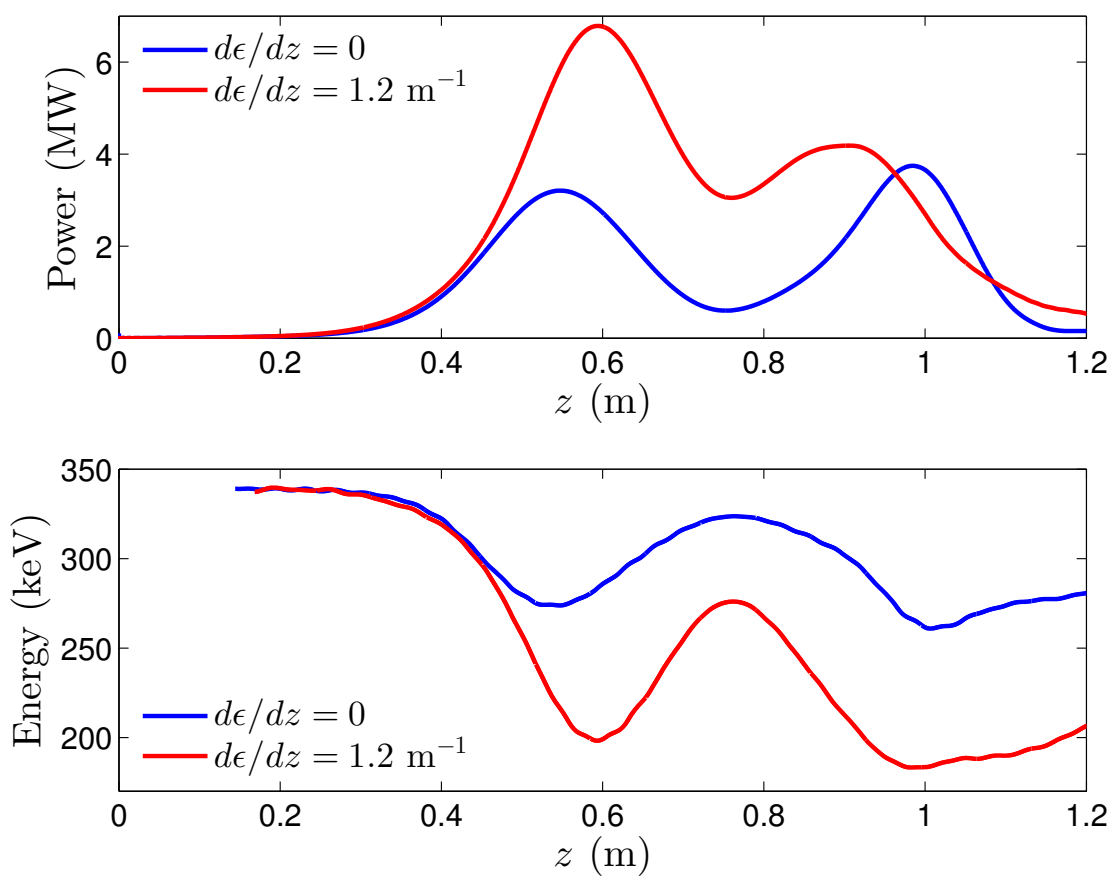

FIG. 16. $\mathrm{TM}_{01}$ power and beam energy loss comparing the "long" taper and un-tapered amplifiers.

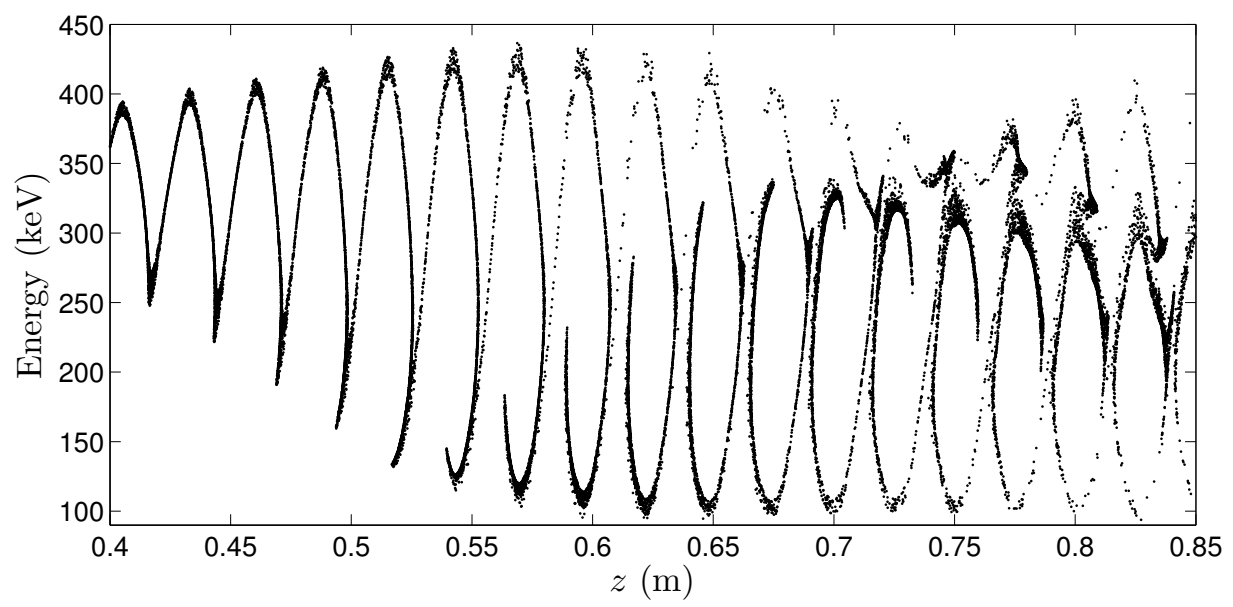

FIG. 17. Longitudinal phase space showing onset of beam trapping and synchrotron oscillations. 

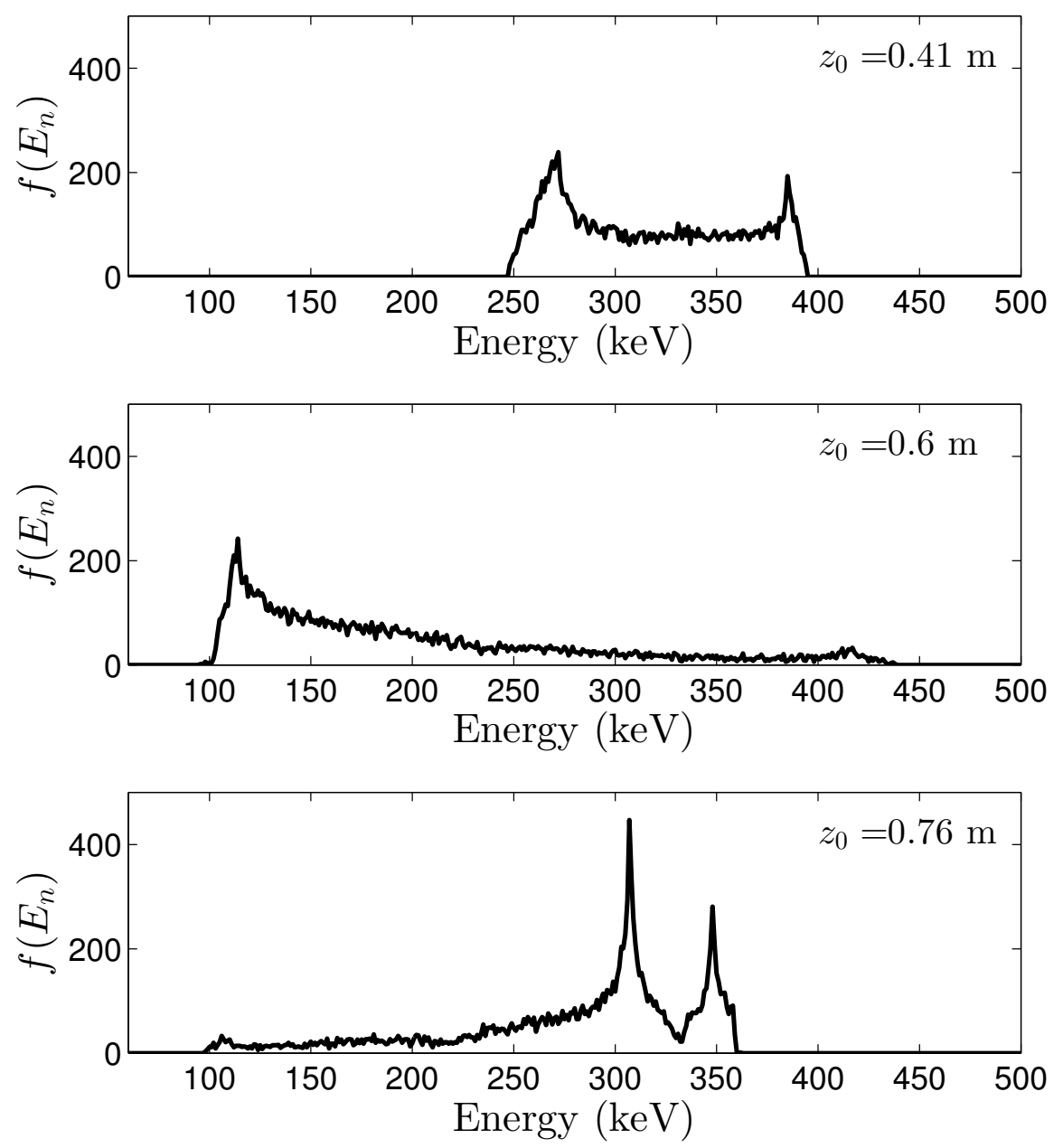

FIG. 18. Evolution of energy distribution. The bin sizes in energy are $1 \mathrm{keV}$. The thickness of the beam slice for sampled particles in the energy distribution was $2 \pi / k_{z}\left(z_{0}\right)$ centered at $z_{0}$. 

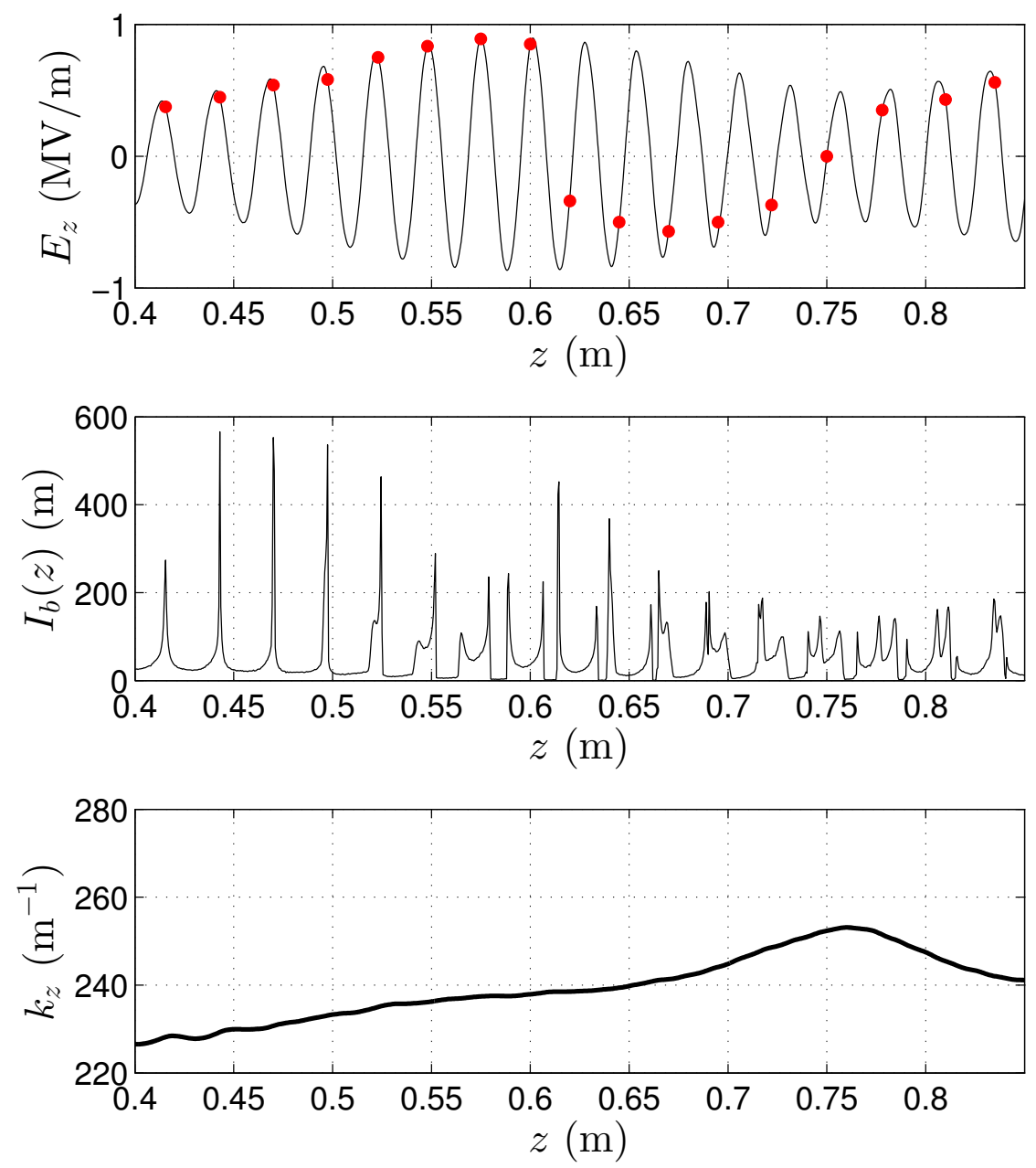

FIG. 19. Axial electric field, beam bunching showing relative phasing between field and bunches and evolution of axial wave number, $k_{z}$. 


\section{Linear Tapers Along a Section of the Interaction Region("Short" taper)}

As seen in the previous section there is little benefit to starting the taper too close to the beginning of the amplifier due to potential detuning of the instability. We next consider a taper only in the region where the beam is close to saturation in the un-tapered source. In this case, the relative permittivity remains constant at $\epsilon_{i}=4$ for $z \leq L_{i}$, and $\epsilon=\epsilon_{f}$ for $z \geq L_{f}$. The tapered dielectric was simulated in OOPIC as described in the previous section, and simulations were run for several values of $\epsilon_{f}$ ranging from 5 to 7 . In all the short taper simulations, $L_{i}=0.3 \mathrm{~m}$ and $L_{f}=1.2 \mathrm{~m}$. Figure 20 summarizes the results of the "short" taper simulations showing the microwave power output at the first and second synchrotron oscillation peaks. In this case, the microwave power at the second synchrotron peak is always larger than at the first, indicating improved energy exchange throughout the synchrotron oscillations. Figure 21 shows a maximum saturated power of $8.1 \mathrm{MW}$ at $z=0.82 \mathrm{~m}$ for the best performing "short" taper with $\epsilon_{f}=6.25$. Here the beam energy at saturation is approximately $165 \mathrm{keV}$ yielding an amplifier efficiency of $48 \%$ compared to $18 \%$ for the un-tapered amplifier and $40 \%$ for the best performing "long" taper. Also note that, compared to the best "long" taper case shown in Fig. 16, the location of both the power maxima has been shifted forward, and closer together, in the best "short" taper case. The longitudinal phase space shown in Fig. 22 shows a substantially larger population of trapped electrons as the beam starts to execute synchrotron oscillations near $z=0.58 \mathrm{~m}$. It is also evident, that when entering the second synchrotron oscillation that the energy of trapped electrons falls to a value significantly lower than at the first saturation peak. This is also apparent in the energy distributions shown in Fig. 23 where at the first saturation peak, the main population of electrons are between energies of approximately $125 \mathrm{keV}$ to $250 \mathrm{keV}$ at $z=0.58 \mathrm{~m}$ and at the second saturation peak at approximately $z=0.82 \mathrm{~m}$ the trapped electron population is between $75 \mathrm{keV}$ and $125 \mathrm{keV}$. Also, the trapped electron population is much more "localized" in energy than in either the un-tapered source, or the source with the "long" taper. This is consistent with the behavior shown in Fig. 24 which shows that the electron bunches remain in a decelerating phase of the wave over a larger cumulative region of the amplifier. In fact, the electron bunches are only in an accelerating phase of the wave

prior to the second saturation peak for $0.6 \mathrm{~m} \leq z \leq 0.7 \mathrm{~m}$. Also, after the microwave field reaches a minimum at approximately $z=0.68 \mathrm{~m}$ the wave number $k_{z}$ continues to increase 


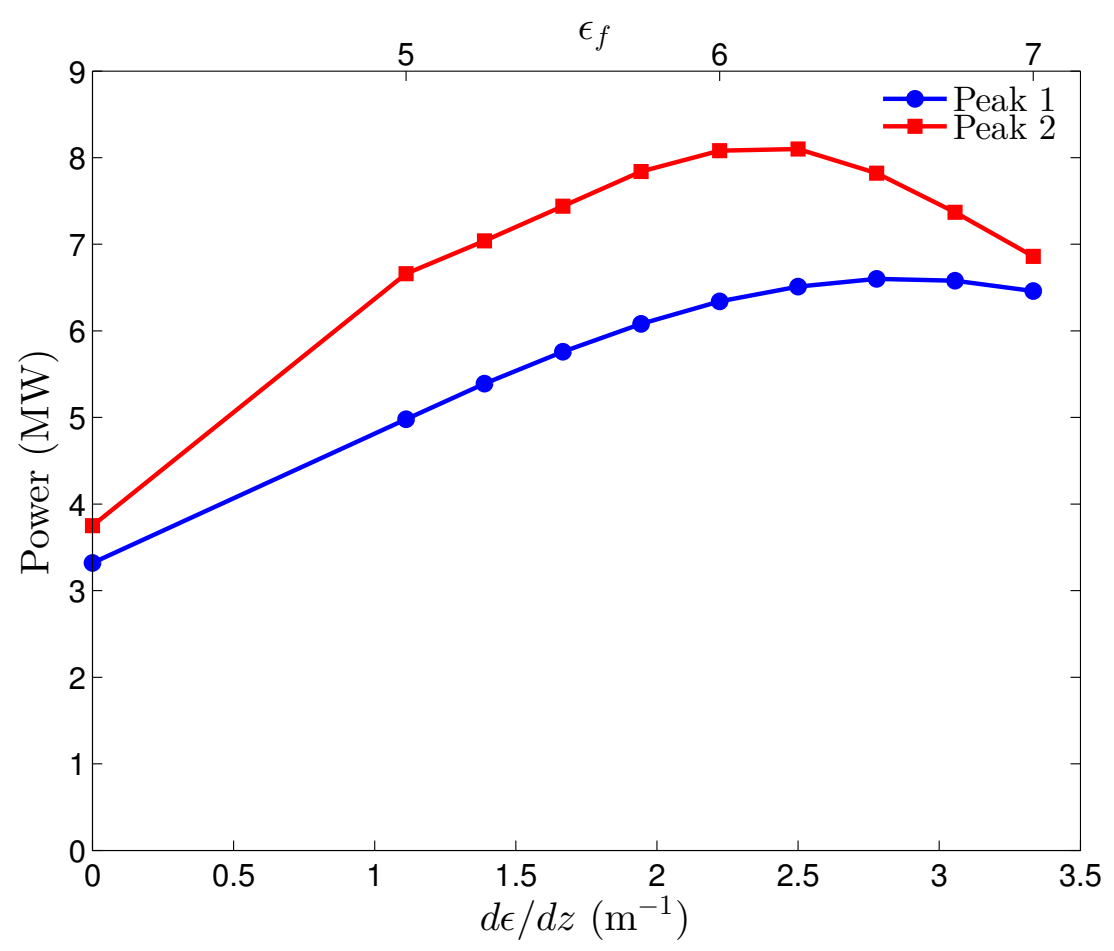

FIG. 20. Power output at first and second synchrotron oscillation peak for a Cerenkov amplifier for the "short" taper.

allowing the phase velocity to decrease maintaining improved synchronism up to the second saturation peak at $z=0.82 \mathrm{~m}$. 

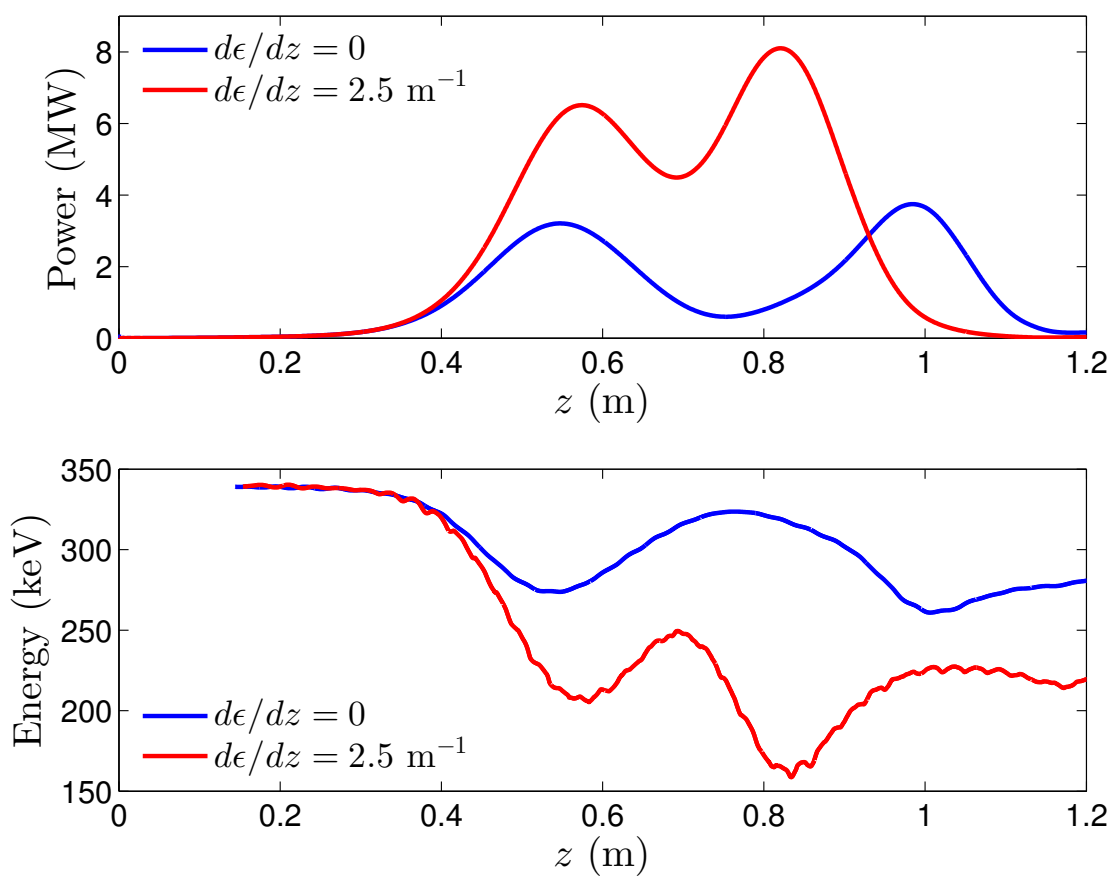

FIG. 21. TM 01 power and beam energy loss comparing the "short" taper and un-tapered amplifiers.

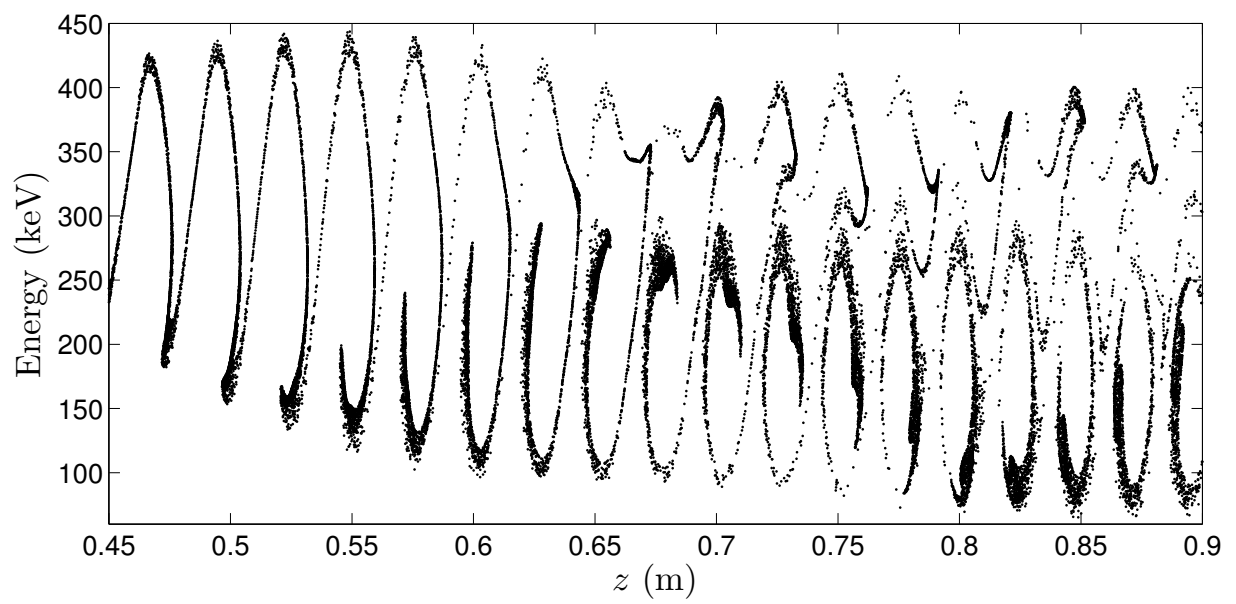

FIG. 22. Longitudinal phase space showing onset of beam trapping and synchrotron oscillations. 

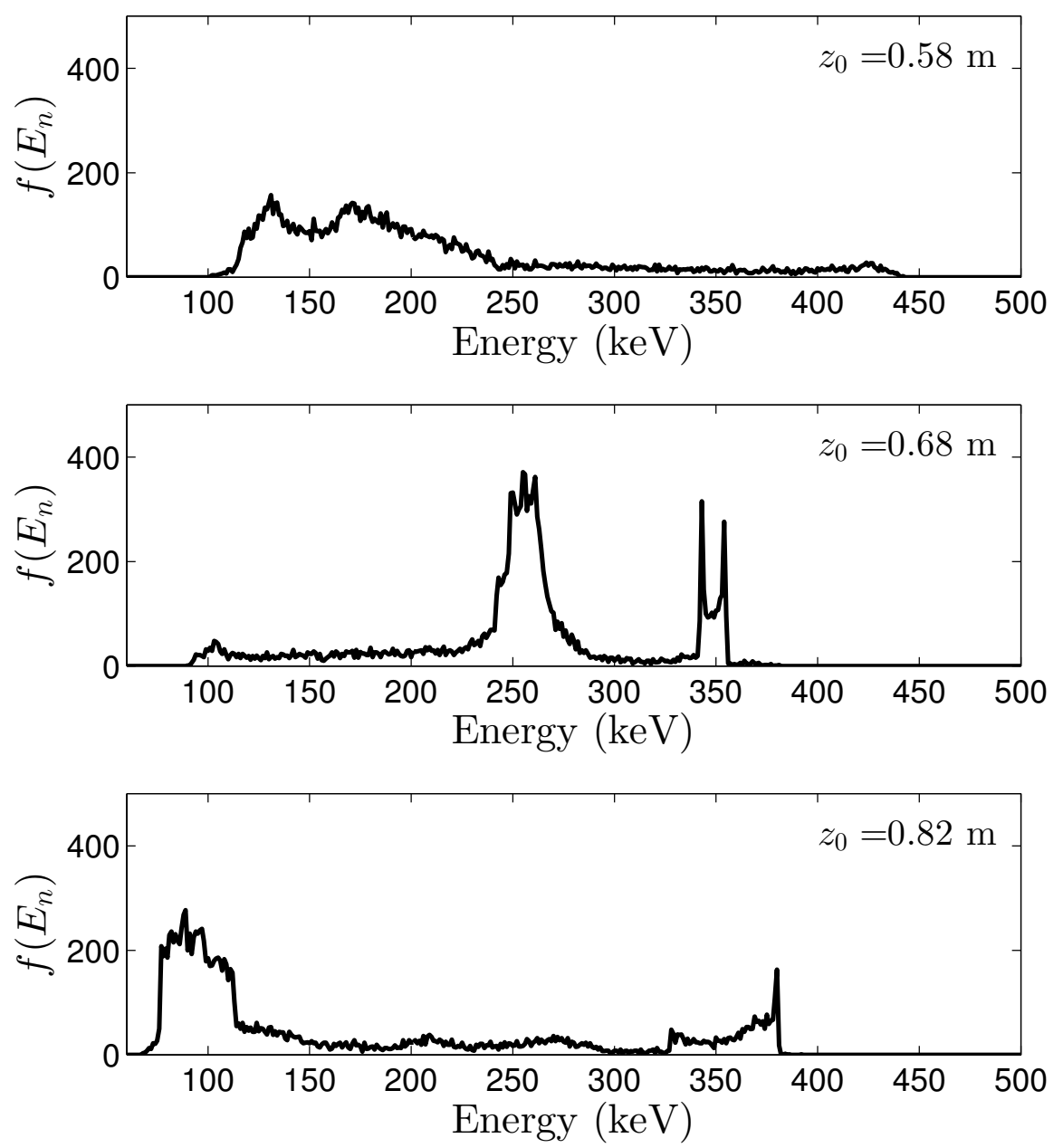

FIG. 23. Evolution of energy distribution. The bin sizes in energy are $1 \mathrm{keV}$. The thickness of the beam slice for sampled particles in the energy distribution was $2 \pi / k_{z}\left(z_{0}\right)$ centered at $z_{0}$. 

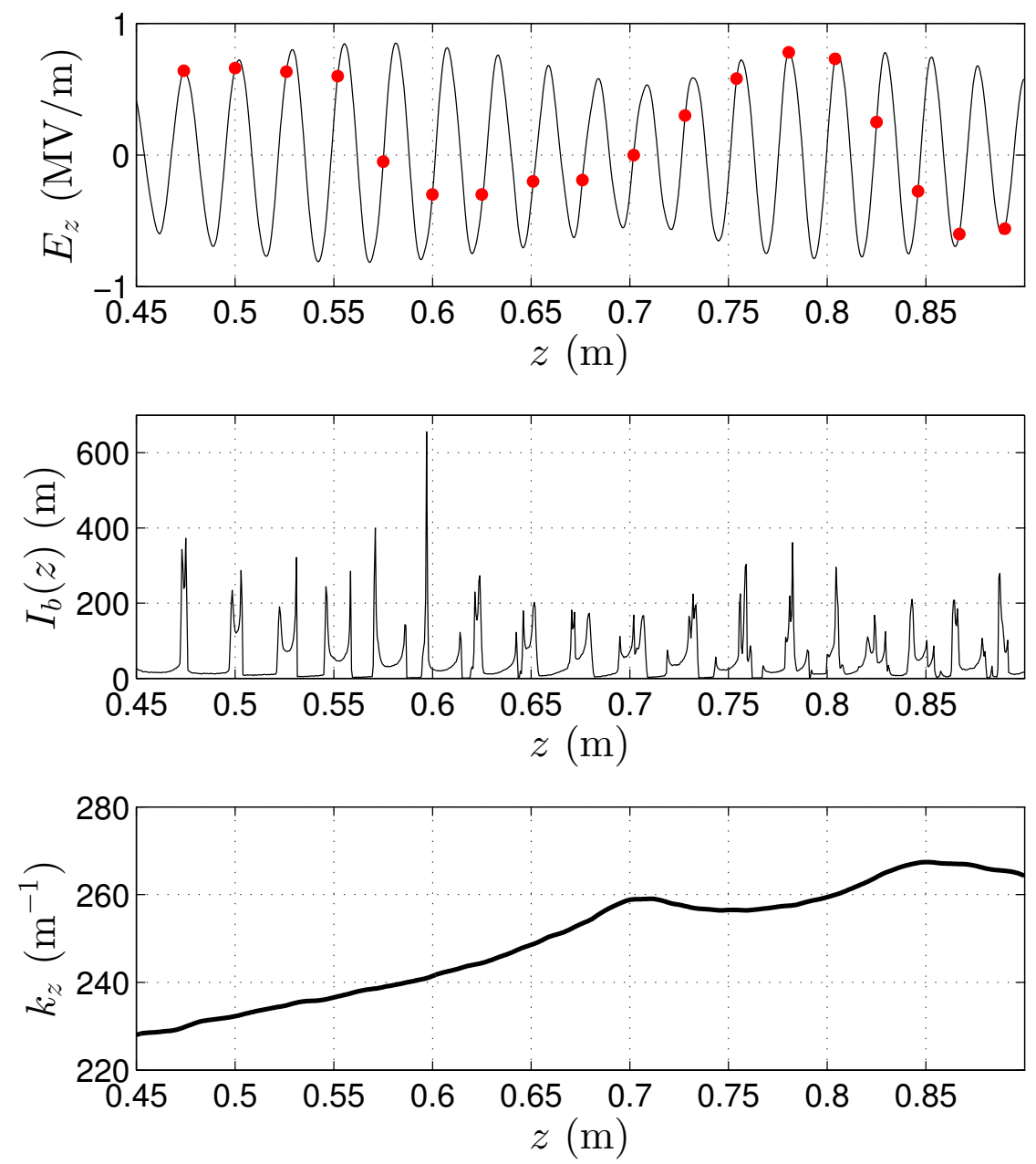

FIG. 24. Axial electric field, beam bunching showing relative phasing between field and bunches and evolution of axial wave number, $k_{z}$. 


\section{CONCLUSION}

Using PIC simulations, we have examined the use of linear tapers in dielectric constant to enhance the efficiency of a Cerenkov microwave source. Two types of tapers were examined: a linear taper over the entire length of the microwave source, and a short taper located near the region when the beam starts to execute synchrotron oscillations. The interaction was investigated using both the electromagnetic wave properties and the details of the beam's longitudinal phase space. Hilbert transforms were employed on the electromagnetic wave field to determine localized amplitude and phase properties to evaluate power and local wave number $k_{z}$. Particle binning in position and phase space were used to evaluate the evolution of energy distributions. Accurate tracking of the electron bunches with respect to the phase of the electromagnetic wave were used to determine when the bunches were in either a decelerating (increasing microwave field) or accelerating (decreasing microwave field) phase of the wave. It was found that the "long" taper over the entire interaction length provided a significant improvement in device efficiency. However, due to localized detuning of the instability, further improvement could be obtained by proper placement of a "short" linear taper near the region of saturation. These results suggest, that by significant micromanagement of the dielectric taper properties further improvements in device performance can be achieved. 


\section{Appendix A: Dispersion Relation}

Expressions for the radial electric fields are evaluated using Eqs. (9)-(14) and are given by

$$
\begin{array}{lrl}
E_{r}(r)=-\frac{i k_{z}}{\kappa} E_{1} I_{1}(\kappa r), & 0 \leq r \leq R_{b 1}, \\
E_{r}(r)=-\frac{i k_{z} Q}{\kappa^{2}}\left[E_{2} I_{1}(Q r)-E_{3} K_{1}(Q r)\right], & R_{b 1} \leq r \leq R_{b 2}, \\
E_{r}(r)=-\frac{i k_{z}}{\kappa}\left[E_{4} I_{1}(\kappa r)-E_{5} K_{1}(\kappa r)\right], & R_{b 2} \leq r \leq R_{d}, \\
E_{r}(r)=-\frac{i k_{z}}{\kappa_{\epsilon}}\left[E_{6} J_{1}\left(\kappa_{\epsilon} r\right)+E_{7} Y_{1}\left(\kappa_{\epsilon} r\right)\right], & R_{d} \leq r \leq R_{0} .
\end{array}
$$

Boundary conditions for the continuity of the axial electric fields at the transverse boundaries are given by

$$
\begin{aligned}
E_{1} I_{0}\left(\kappa R_{b 1}\right) & =E_{2} I_{0}\left(Q R_{b 1}\right)+E_{3} K_{0}\left(Q R_{b 1}\right) \\
E_{2} I_{0}\left(Q R_{b 2}\right)+E_{3} K_{0}\left(Q R_{b 2}\right) & =E_{4} I_{0}\left(\kappa R_{b 2}\right)+E_{5} K_{0}\left(\kappa R_{b 2}\right) \\
E_{4} I_{0}\left(\kappa R_{d}\right)+E_{5} K_{0}\left(\kappa R_{d}\right) & =E_{6} J_{0}\left(\kappa_{\epsilon} R_{d}\right)+E_{7} Y_{0}\left(\kappa_{\epsilon} R_{d}\right) \\
E_{6} J_{0}\left(\kappa R_{0}\right)+E_{7} Y_{0}\left(\kappa R_{0}\right) & =0
\end{aligned}
$$

Similarly, boundary conditions for the continuity of radial electric displacement are given by

$$
\begin{aligned}
\left.E_{1} I_{1}\left(\kappa R_{b 1}\right)\right) & =\frac{Q}{\kappa}\left[E_{2} I_{1}\left(Q R_{b 1}\right)-E_{3} K_{1}\left(Q R_{b 1}\right)\right] \\
\frac{Q}{\kappa}\left[E_{2} I_{1}\left(Q R_{b 2}\right)-E_{3} K_{1}\left(Q R_{b 2}\right)\right] & =\left[E_{4} I_{1}\left(\kappa R_{b 2}\right)-E_{5} K_{1}\left(\kappa R_{b 2}\right)\right] \\
\frac{1}{\kappa}\left[E_{4} I_{1}\left(\kappa R_{d}\right)-E_{5} K_{1}\left(\kappa R_{d}\right)\right] & =\frac{\epsilon}{\kappa_{\epsilon}}\left[E_{6} J_{1}\left(\kappa_{\epsilon} R_{d}\right)+E_{7} Y_{1}\left(\kappa_{\epsilon} R_{d}\right)\right]
\end{aligned}
$$

Eqs. (A5)-(A11) constitute a set of equations defined by

$$
\mathbf{D}\left(k_{z}, \omega\right) \cdot \mathbf{E}=0,
$$

where $\mathbf{E}$ constitutes the various electric field coefficients and where Eq. (A12) has a nontrivial solution if

$$
\operatorname{det}\left[\mathbf{D}\left(k_{z}, \omega\right)\right]=0 .
$$

The matrix elements of $\mathbf{D}\left(k_{z}, \omega\right)$ are given by

$$
D_{11}=\frac{1}{\kappa} I_{1}\left(\kappa R_{b 1}\right),
$$




$$
\begin{aligned}
& D_{12}=-\frac{Q}{\kappa^{2}} I_{1}\left(Q R_{b 1}\right), \\
& D_{13}=\frac{Q}{\kappa^{2}} K_{1}\left(Q R_{b 1}\right) \text {, } \\
& D_{22}=\frac{Q}{\kappa^{2}} I_{1}\left(Q R_{b 2}\right), \\
& D_{23}=-\frac{Q}{\kappa^{2}} K_{1}\left(Q R_{b 2}\right) \text {, } \\
& D_{24}=-\frac{1}{\kappa} I_{1}\left(\kappa R_{b 2}\right) \text {, } \\
& D_{25}=\frac{1}{\kappa^{2}} K_{1}\left(\kappa R_{b 2}\right) \text {, } \\
& D_{34}=\frac{1}{\kappa} I_{1}\left(\kappa R_{d}\right), \\
& D_{35}=-\frac{1}{\kappa} K_{1}\left(\kappa R_{d}\right) \text {, } \\
& D_{36}=-\frac{\epsilon}{\kappa} J_{1}\left(\kappa_{\epsilon} R_{d}\right) \text {, } \\
& D_{37}=-\frac{\epsilon}{\kappa_{\epsilon}} Y_{1}\left(\kappa_{\epsilon} R_{d}\right) \text {, } \\
& D_{41}=I_{0}\left(\kappa R_{b 1}\right) \text {, } \\
& D_{42}=-I_{0}\left(Q R_{b 1}\right), \\
& D_{43}=-K_{0}\left(Q R_{b 1}\right), \\
& D_{52}=I_{0}\left(Q R_{b 2}\right), \\
& D_{53}=K_{0}\left(Q R_{b 2}\right) \text {, } \\
& D_{54}=-I_{0}\left(\kappa R_{b 2}\right) \text {, } \\
& D_{55}=-K_{0}\left(\kappa R_{b 2}\right) \text {, } \\
& D_{64}=I_{0}\left(\kappa R_{d}\right), \\
& D_{65}=K_{0}\left(\kappa R_{d}\right) \text {, } \\
& D_{66}=-J_{0}\left(\kappa_{\epsilon} R_{d}\right), \\
& D_{67}=-Y_{0}\left(\kappa_{\epsilon} R_{d}\right) \text {, } \\
& D_{76}=J_{0}\left(\kappa_{\epsilon} R_{0}\right) \text {, } \\
& D_{77}=Y_{0}\left(\kappa_{\epsilon} R_{0}\right),
\end{aligned}
$$

with all remaining matrix elements being zero. Eq. (A13) is a complicated transcendental equation in $k_{z}$ and $\omega$ which can be solved numerically to determine the dispersion relation $k_{z}=k_{z}(\omega)$. In addition, the values of $E_{1}$ thru $E_{7}$ can be determined within a constant from the null space of Eq. (A12) which is used to evaluate the mode structure. 


\section{ACKNOWLEDGMENTS}

This work was performed under the auspices of the U.S. Department of Energy by Lawrence Livermore National Laboratory under Contract DE-AC52-07NA27344.

\section{REFERENCES}

${ }^{1}$ V. L. Ginsburg, Dokl. Akad. Nauk. 56, 253 (1947).

2 J. V. Jelley, "Cerenkov radiation," (Pergamon Press, New York, 1958) pp. 44-51.

${ }^{3} \mathrm{H}$. Lashinsky, in Advances in Electronics and Electron Physics, Vol. 14, edited by L. Martin (Academic Press, New York, 1961) pp. 265-297.

${ }^{4}$ J. E. Walsh, T. C. Marshall, and S. P. Schlesinger, Phys. Fluids 20, 709 (1977).

${ }^{5}$ K. L. Felch, K. O. Busby, R. W. Layman, D. Kapilow, and J. E. Walsh, Appl. Phys. Lett. 38, 601 (1981).

${ }^{6}$ H. P. Freund and A. K. Ganguly, Phys. Fluids B 2, 2506 (1990).

${ }^{7}$ B. R. Poole, D. T. Blackfield, and J. F. Camacho, Phys. Rev. ST Accel. Beams 12, 080705 (2009).

${ }^{8}$ J. R. Harris, K. L. Ferguson, J. W. Lewellen, S. P. Niles, B. Rusnak, R. L. Swent, W. B. Colson, T. I. Smith, C. H. Boulware, T. L. Grimm, P. R. Cunningham, M. S. Curtin, D. C. Miccolis, D. J. Sox, and W. S. Graves, Phys. Rev. ST Accel. Beams 14, 053501 (2011).

${ }^{9}$ B. R. Poole and J. R. Harris, in Proceedings of 2011 Particle Accelerator Conference (2011) pp. 1831-1833.

${ }^{10}$ T. C. Marshall and T. B. Zhang, in AIP Conference Proceedings, Vol. 396 (1997) pp. $105-119$.

${ }^{11}$ T. C. Marshall, in AIP Conference Proceedings, Vol. 335 (1995) pp. 261-270.

${ }^{12}$ L. Schächter, Phys. Rev. A 43, 3785 (2009).

${ }^{13}$ L. Schächter and J. A. Nation, in Proceedings SPIE, Vol. 1407 (1991) p. 44.

${ }^{14}$ A. Hirata and T. Shiozawa, J. Appl. Phys. 82, 5907 (1997).

${ }^{15}$ D. Shiffler, J. Luginsland, D. M. French, and J. Watrous, IEEE Trans. Plasma Sci. 38, $1462(2010)$.

${ }^{16}$ While some authors use the term tapering to refer to a gradual reduction in the thickness of the dielectric liner near its ends in order to minimize reflections, (as in W. Peter and E. 
Garate, Phys. Rev. A 45, 8833 (1992)), we will exclusively use the term to refer to changes in the dielectric liner which are used to maintain synchronism as energy is extracted from the beam.

${ }^{17}$ B. W. Gore, V. B. Asgekar, and A. Sen, Physica Scripta 53, 62 (1996).

${ }^{18} \mathrm{P}$. van der Slot, I. de la Fuente, and K. Boller, in Proceeding of the 2004 FEL Conference (2004) pp. 53-56.

${ }^{19}$ T. Shiozawa and T. Nishimura, Appl. Phys. Lett. 68, 1443 (1996).

${ }^{20}$ T. Shiozawa, H. Takahashi, and Y. Kimura, IEEE J. Quant. Electronics 32, 2037 (1996).

${ }^{21}$ S. J. Smith and E. M. Purcell, Phys. Rev. 92, 1069 (1953).

${ }^{22}$ T. Shiozawa and T. Yoshitake, IEEE J. Quant. Electronics 31, 539 (1995).

${ }^{23}$ T. J. Orzechowski, B. R. Anderson, J. C. Clark, W. M. Fawley, A. C. Paul, D. Prosnitz, E. T. Scharlemann, S. M. Yarema, D. B. Hopkins, A. M. Sessler, and J. S. Wurtele, Phys. Rev. Lett. 57, 2172 (1986).

${ }^{24}$ A. Christodoulou, D. Lampiris, K. Polykandriotis, W. B. Colson, P. P. Crooker, S. Benson, J. Gubeli, and G. R. Neil, Phys. Rev. E 66, 056502 (2002).

${ }^{25}$ W. B. Colson and R. D. McGinnis, Nucl. Instrum. and Methods A 445, 49 (2000).

${ }^{26}$ OOPIC is a product of TECH-X, Boulder, Colorado. 